\title{
Using rules to screen for cooperative types: rule- following and restraint in common pool resource systems
}

Citation for published version (APA):

Kimbrough, E. O., \& Vostroknutov, A. (2012). Using rules to screen for cooperative types: rule-following and restraint in common pool resource systems. METEOR, Maastricht University School of Business and Economics. METEOR Research Memorandum No. 054 https://doi.org/10.26481/umamet.2012054

Document status and date:

Published: 01/01/2012

DOI:

10.26481/umamet.2012054

Document Version:

Publisher's PDF, also known as Version of record

Please check the document version of this publication:

- A submitted manuscript is the version of the article upon submission and before peer-review. There can be important differences between the submitted version and the official published version of record.

People interested in the research are advised to contact the author for the final version of the publication, or visit the DOI to the publisher's website.

- The final author version and the galley proof are versions of the publication after peer review.

- The final published version features the final layout of the paper including the volume, issue and page numbers.

Link to publication

\footnotetext{
General rights rights.

- You may freely distribute the URL identifying the publication in the public portal. please follow below link for the End User Agreement:

www.umlib.nl/taverne-license

Take down policy

If you believe that this document breaches copyright please contact us at:

repository@maastrichtuniversity.nl

providing details and we will investigate your claim.
}

Copyright and moral rights for the publications made accessible in the public portal are retained by the authors and/or other copyright owners and it is a condition of accessing publications that users recognise and abide by the legal requirements associated with these

- Users may download and print one copy of any publication from the public portal for the purpose of private study or research.

- You may not further distribute the material or use it for any profit-making activity or commercial gain

If the publication is distributed under the terms of Article $25 \mathrm{fa}$ of the Dutch Copyright Act, indicated by the "Taverne" license above, 
Erik O. Kimbrough,

Alexander Vostroknutov

Using Rules to Screen for Cooperative Types:

Rule-Following and Restraint in Common Pool Resource Systems

$\mathrm{RM} / 12 / 054$

\section{METEOR}

Maastricht University school of Business and Economics

Maastricht Research School of Economics

of Technology and Organization

\section{PO. Box 616}

NL - 6200 MD Maastricht

The Netherlands 


\title{
Using Rules to Screen for Cooperative Types: Rule-Following and Restraint in Common Pool Resource Systems*
}

\author{
Erik O. Kimbrough $^{\dagger} \quad$ Alexander Vostroknutov ${ }^{\ddagger}$
}

\author{
August 2012 \\ Draft. Do not cite without authors' permission.
}

\begin{abstract}
We argue that rules and rule-following provide a key means of solving social dilemmas by revealing information about individual willingness to cooperate, thereby facilitating assortative matching and the exclusion of non-cooperative types. Rules impose costs on prospective entrants to any group and ensure that only those willing to pay such costs will join. To illustrate this point, we study a novel, repeated common pool resource game in which current resource stocks depend on resource extraction in previous periods. We show that for a sufficiently high regrowth rate, there is no commons dilemma: the resource will be preserved indefinitely in equilibrium. Behavioral tests of the model indicate that favorable ecological characteristics are necessary but insufficient to encourage effective CPR governance. However, by screening and sorting individuals according to their willingness to follow costly rules in an individual choice task, we show that CPR groups composed of rule-followers are less likely to exhaust the resource than both groups of rule-breakers and mixed-type groups.
\end{abstract}

JEL classifications: $C 9, C 7, D 7$

Keywords: common pool resources, rule-following, experimental economics

\footnotetext{
${ }^{*}$ We would like to thank Taylor Jaworski, Fabio Méndez, Alexander Smith, and seminar participants at the University of Arkansas, the 2012 Canadian Economic Association Annual Conference in Calgary, and the 2012 Economic Science Association International conference in New York for helpful comments.

${ }^{\dagger}$ Department of Economics, Simon Fraser University, 8888 University Drive, Vancouver, BC V5A 1S6, Canada, e-mail: ekimbrou@sfu.ca

ҒCorresponding Author: Department of Economics, Maastricht University, P.O. Box 616, Maastricht 6200 MD, The Netherlands. e-mail: a.vostroknutov@maastrichtuniversity.nl
} 


\section{Introduction}

Often the justification for any rule is sought in its value in solving some particular problem. Thus, in most countries, motorists drive on the right side of the road to solve a potentially deadly coordination problem, and societies impose punishments for theft and other crimes to increase the relative costs of undesirable actions. We argue that the plethora of social rules also serve a more subtle purpose: by observing whether an individual adheres to some rule, we readily gain information about that individual's willingness to cooperate. By excluding those individuals unwilling to follow rules, groups screen out non-cooperators and are able to reap the benefits of cooperation. Thus, the constant presence of rules in our daily lives and the ready observability of others' adherence to them together facilitate the resolution of social dilemmas, which suggests an additional fundamental reason for the prevalence of and high esteem for explicit (occasionally arbitrary) rules at every level of social organization. Thus, any estimate of the social value of rules, which would typically consider their instrumental value in solving particular coordination problems, should also take into account their value as screening mechanisms for cooperative types.

We illustrate the screening function of rules by studying a novel common pool resource (CPR) problem. CPR management is fraught with incentive issues. The classic statement of the problem highlights the tension between individual and social incentives for maintaining the stock of a renewable resource in the absence of welldefined property rights (Gordon, 1954; Hardin, 1968). Thus when property rights are ill-defined, resources will be depleted because individuals lack incentives for preservation. In her seminal book, Elinor Ostrom (1990) provides vivid accounts of common pool resource management in a variety of communities around the world. Intriguingly, she observes great diversity in resource governance outcomes: while some communities are able to sustain common resources for hundreds of years, through changes in government, wars and natural disasters, others consistently fail despite many attempts to design the appropriate governance institutions. ${ }^{1}$

To understand the sources of this diversity, Ostrom has proposed that CPRs be understood as "social-ecological systems" in which multiple interacting factors influence successful resource governance (e.g. Ostrom, 2009). In general, these can be grouped into two categories: 1) ecological characteristics of the resource system, like the speed of re-growth of the resource or the number of users, and 2) social and behav-

\footnotetext{
${ }^{1}$ Among examples of successful sustainability of CPRs are huerta irrigation institutions in Spain and common grazing fields and forests in the Swiss Alps. Among failures are fisheries in Turkey, Canada and Sri Lanka as well as water management in some counties in South California.
} 
ioral factors that influence CPR usage. This paper combines theory and experiment to understand precisely how social and ecological factors interact to influence the sustainability of CPRs.

We develop a novel model of a dynamic, multi-player common pool resource game that captures the effects of crucial ecological parameters on resource dynamics. In our game individuals allocate productive efforts between a private, inexhaustible outside option and an exhaustible common pool resource that yields larger per-unit returns to effort. We solve for the Subgame Perfect Nash equilibrium and show that the optimal resource extraction strategy depends on the rate of resource regeneration. In a finitely repeated game, for a sufficiently high regeneration rate, individuals maintain the resource indefinitely in equilibrium. Moreover, this strategy corresponds to the Social Planner's solution of the game. Thus, for a broad range of parameter configurations, stocks of common pool resources will be preserved in equilibrium, and there is no commons dilemma. However, below some threshold rate, individuals will instead exhaust the resource, while a social planner would prefer to preserve the resource indefinitely. The dependence of equilibrium behavior on ecological parameters of the model helps explain the prevalence of both successful and unsuccessful management of common pool resources identified around the world (Ostrom, 1990, 2010).

To test our model of the CPR game, we report a series of laboratory experiments. Well-known laboratory results in common pool resource environments suggest that, when "individuals do not know one another, cannot communicate effectively, and thus cannot develop agreements, norms, and sanctions," groups are prone to overharvest resources in a manner broadly aligned with the classic statement of the common pool resource problem (Ostrom, Gardner, and Walker, 1994, p. 319). However, our model predicts that under some sets of parameters, groups of anonymous, socially separated individuals will nevertheless avoid overharvesting and maintain the resource indefinitely. Thus, we can test experimentally whether people respond to different ecological parameters of the CPR system in the way predicted by our model.

As Ostrom (2010) notes, all CPR systems are inextricably bound to the social systems in which they are managed and exploited, and thus favorable ecological characteristics may be necessary conditions without being sufficient to ensure successful CPR management. It is an open question whether Nash equilibrium will provide an accurate depiction of behavior in dynamic CPR systems, and we have many reasons to believe that observed behavior in an experimental implementation of the model may deviate substantially from Nash predictions. Previous studies of social 
dilemmas report considerable behavioral heterogeneity, both across countries and across individuals within a given country (e.g. Henrich et al. 2005, 2010; Gächter, Herrmann, and Thöni, 2010; Herrmann, Thöni, and Gächter, 2008; Fischbacher and Gächter, 2010).

Following Smith (2003), one way of understanding observed heterogeneity in social dilemmas is to conceive of various player types, where a player's type can be inferred from her actions. Previous research has demonstrated the efficacy of this method, both that it is possible to identify consistent player types on the basis of observed play (e.g. Fischbacher, Gächter, and Fehr, 2001; McCabe, Houser, Ryan, Smith, and Trouard, 2001; Houser, Keane, and McCabe, 2004; Kurzban and Houser, 2005) and that sorting by type can encourage cooperation among the positively assorted (e.g. Gunnthorsdottir, Houser, and McCabe, 2007; Rigdon, McCabe, and Smith, 2007; Wilson, Jaworski, Schurter, and Smyth, 2012). However, in previous research, types were identified by observing play in the relevant game. From the point of view of encouraging cooperation, it would be preferable to infer information about a player's type prior to observing play in the game-by then it may be too late.

With this in mind, our experiments allow us to identify a particular behavioral trait, which we argue characterizes players' types in social dilemmas, and which may influence whether and/or how the dynamics of CPR usage diverge from the equilibrium predictions. Specifically, following Kimbrough and Vostroknutov (2012), we identify types with an unrelated experimental task in which subjects are asked to follow an arbitrary and costly rule. We identify our subjects as either rule-followers or rule-breakers to test the hypothesis that rule-followers will be more inclined to preserve the CPR than rule-breakers.

This idea has precedent in the economics of religion where Iannaccone (1992) also makes the connection between rule following and cooperation. He argues that costly rules imposed by religious communities on prospective members (e.g. dietary restrictions) serve as screening devices to deter entry by free-riders and promote cooperation within the community. Similar behavioral patterns have been implicated in field studies of successful CPR systems, where rule-abiding individuals are able to sustain CPRs. For example, Ostrom (1990) reports very little cheating and resource overuse in communities that managed to sustain a common resource for many years. People in such communities do not overharvest the resource even when monitoring is nearly non-existent and there are huge benefits from overusage. ${ }^{2}$ Similarly, Tang

\footnotetext{
${ }^{2}$ For example, court records from one year in the 15th century Spain show that for 25,000 estimated opportunities of water theft only 200 cases took place which gives the minuscule infraction rate of
} 
(1994, p.229) shows that groups of individuals with a propensity to follow rules are much more successful in sustaining their resources than groups without such propensities.

These findings hint at the importance of assortative matching and the exclusion of defectors to the successful management of CPRs, but in field studies it is difficult to distinguish successful CPR management due to groups composed of cooperative types from successes due to ecological factors. Here we report laboratory experiments in which we can measure and control the type-composition of each CPR system: by measuring rule-following proclivities of each subject, we can compare the behavior of rule-followers and rule-breakers in a mixed population and we can assortatively match subjects into groups with others of the same type to identify the impact of group composition. In particular, in two treatments, we observe individuals' rulefollowing decision prior to their play in the CPR game, and we assortatively match them without their knowledge into groups composed of similar individuals. In two other treatments, individuals first play the CPR game in randomly assigned groups and then we observe their willingness to follow the costly rule.

Ultimately, our results suggest an intimate relationship between ecological and social factors in preserving CPRs. While favorable ecological conditions are necessary for CPR sustainability, they are not sufficient in our behavioral experiments. Even under favorable ecological conditions, when groups do not screen out non-cooperative (rule-breaking) types, they may be unable to preserve CPRs. Our experiment reveals that such screening can by performed by observing the willingness of prospective entrants to follow rules and excluding those who are revealed to be unwilling. Thus,

The paper is organized as follows. In Section 2 we describe the CPR game and the Subgame Perfect Nash equilibrium (Section 2.1: one-shot stage game and its NE; Section 2.2: finitely repeated game and its SPNE; Section 2.3: solution to the Social Planner's problem), and in Section 2.4 we discuss our model in relation to other models of CPRs. Section 3 describes the experimental design. Section 4 reports the results of our experiments, and section 5 offers some concluding remarks. Appendices contain proofs of the propositions, lemmata, the Social Planner's problem with and without a negative externality, description of variables used in the statistical analysis, additional experimental findings, and experimental instructions.

0.008. This is given that monitors could only check each household approximately once a year (Ostrom, 1990, p. 75). 


\section{The Common Pool Resource Game}

In this section we present a formal model of the Common Pool Resource (hereafter $\mathrm{CPR}$ ) game to highlight how the equilibrium resource extraction strategy depends on ecological parameters.

\subsection{One-Shot Game}

We start with a description of the one-shot stage game. Suppose there is a resource stock $w>0$. There are $n$ players who simultaneously choose how much effort to exert in harvesting the resource. Each player $i^{\prime}$ s chooses effort $e_{i} \in[0, \bar{e}]$ where $\bar{e}>0$ is a common maximum effort level that can be exerted by each player (e.g. hours worked per day with constant productivity). Let $E=\sum_{i=1 . . n} e_{i}$ denote the sum of all efforts. The payoff function for player $i$ is

$$
\pi_{i}\left(e_{i}, e_{-i} \mid w\right)= \begin{cases}\bar{e}-e_{i}+\alpha e_{i} & \text { if } w-E>0 \\ \bar{e}-e_{i}+\alpha \frac{w}{n} & \text { if } w-E \leq 0\end{cases}
$$

The intuition behind $\pi_{i}$ is the following. For simplicity we assume that the amount of the resource, efforts, and players' utilities are all denominated in the same units. If the sum of all efforts $E$ does not exceed the available amount of the resource $w$ then each player $i$ receives a return on effort equal to $\alpha e_{i}$, where $\alpha>1$ ensures that players find it worthwhile to exert effort harvesting the resource. The payoff in this case is $\bar{e}-e_{i}$, the amount of effort not spent, plus $\alpha e_{i}$, the return from exerted effort, or $\bar{e}+(\alpha-1) e_{i}$. If, however, the sum of harvesting efforts exceeds the resource stock, then all players share the remaining stock equally, and the payoff is $\bar{e}-e_{i}+\alpha \frac{w}{n} .^{3}$ This finalizes the description of the stage game. Hereafter, we will call any such game, parameterized by $w$, a $w$-game.

Proposition 1. If $w>n \bar{e}$ then the unique pure strategy Nash Equilibrium of the w-game is $e_{i}=\bar{e}$ for all $i=1 . . n$. If $w \leq n \bar{e}$ then the unique pure Nash Equilibrium of $w$-game is $e_{i}=\frac{w}{n}$

\footnotetext{
${ }^{3}$ We could also assume that after choosing their efforts, players start "eating" the resource with the same "speed" and continue eating until either all have exerted the chosen effort or the resource is exhausted. In this case, players who choose low efforts still receive $\alpha e_{i}$ if the resource is not fully depleted, and only those who choose high effort end up sharing the remains. Under this alternative specification our results are unchanged.
} 
for all $i=1 . . n$.

Proof. See Appendix A.

\subsection{Finitely Repeated Game}

Next, we analyze the repeated w-game. Suppose that players make simultaneous effort decisions in $L$ consecutive periods. In each period they may choose to exert effort $e_{i}$ up to some maximum effort $\bar{e}$. Moreover, the resource stock in future periods depends on the amount remaining after harvesting decisions made in the current period. We assume that the resource naturally grows at a rate inversely proportional to its current size. Furthermore, assume that due to exogenous environmental or technical factors, there exists a maximum resource capacity $\bar{w}$. Now, suppose that after harvesting in period $t-1$ the remaining resource stock is $w_{t-1}^{*}$. Then, the stock available before period $t$ is $w_{t-1}^{*}+\beta\left(\bar{w}-w_{t-1}^{*}\right) \mathbb{1}_{w_{t-1}^{*} \geq \tau}$. Here $0<\beta<1$ is the growth rate and $\mathbb{1}_{w_{t-1}^{*} \geq \tau}$ equals 1 whenever $w_{t-1}^{*} \geq \tau$ and 0 otherwise. The interpretation is the following. The more depleted the resource $w_{t-1}^{*}$ the faster the resource is able to regenerate. The growth rate approaches 0 as the resource stock approaches $\bar{w}$. However, if the resource is depleted below some level $\tau \ll \bar{e}$, then the resource is exhausted and no regrowth happens thereafter.

Now we can define the repeated $w$-game. Denote by $E_{t}=\sum_{i=1 . . n} e_{i t}$ the sum of efforts of all players in period $t=1$..L. Suppose that before period 1 the stock of the resource is $w_{1}$. In period 1 players choose effort levels that determine $E_{1}$ and, consequently, the resource stock decreases to $w_{1}^{*}=\left(w_{1}-E_{1}\right) \mathbb{1}_{w_{1}-E_{1}>0}$. This is the amount of the resource left after period 1 . Then a portion of the resource regrows, and the stock becomes $w_{2}=w_{1}^{*}+\beta\left(\bar{w}-w_{1}^{*}\right) \mathbb{1}_{w_{1}^{*} \geq \tau}$, which is the amount available at the beginning of period 2. At this moment period 2 begins. Defining the same quantities for all periods we get the resource stock available before period $t>1$ to be

$$
w_{t}=w_{t-1}^{*}+\beta\left(\bar{w}-w_{t-1}^{*}\right) \mathbb{1}_{w_{t-1}^{*} \geq \tau}
$$

where $w_{t}^{*}=\left(w_{t}-E_{t}\right) \mathbb{1}_{w_{t}-E_{t}>0}$.

Let $H_{t}$ denote the set of all histories of effort choices in periods 1 through $t$. The typical element of $H_{t}$ is $h_{t}=\left(\left(e_{i 1}\right)_{i=1 . . n}, \ldots,\left(e_{i t}\right)_{i=1 . . n}\right)$. The history of choices $h_{t}$ defines the sequence $\left(E_{k}\left(h_{t}\right)\right)_{k=1 . . t}$ of sums of efforts. ${ }^{4}$ This sequence in its turn generates the sequence of resource stocks $\left(w_{k}\left(h_{t}\right)\right)_{k=1 . . t}$ available before periods $k$ as determined

\footnotetext{
${ }^{4}$ Here $E_{k}\left(h_{t}\right)$ denotes the sum of efforts in period $k$ as defined by $h_{t}$.
} 
by the recursive application of the resource growth formula above. The stage game played in period $t+1$ after history $h_{t}$ is $\left(w_{t}^{*}+\beta\left(\bar{w}-w_{t}^{*}\right) \mathbb{1}_{w_{t}^{*} \geq \tau}\right)$-game. The utility of player $i$ after terminal history $h_{L}$ is then defined by

$$
\sum_{k=1}^{L} \pi_{i}\left(e_{i t}, e_{-i t} \mid w_{k}\left(h_{L}\right)\right)
$$

This completely defines the (history-dependent) repeated CPR game.

Now we turn to the equilibrium analysis of the finitely repeated CPR game. The following proposition shows that there exists a symmetric Markov Subgame Perfect Nash Equilibrium in which players exert maximum effort $\bar{e}$ in any period in which doing so will not deplete the resource below $\tau$. When the resource stock in period $t$ is $w_{t}<n \bar{e}+\tau$ the equilibrium strategy is for all players to exert effort so that the resource is depleted to exactly $\tau$. Thus, the resource will be preserved indefinitely in equilibrium.

Proposition 2. If $\beta \geq \frac{\tau(n-1)}{\bar{w}-\tau}$ and $\tau \leq \bar{e}$ then the following strategy used by all players constitutes a SPNE. In periods $t=1$..L choose $e_{i t}=\bar{e}$ if the resource before period $t$ is $w_{t} \geq n \bar{e}+\tau$. In period $t=1 . . L-1$ choose $e_{i t}=\frac{w_{t}-\tau}{n}$ if $w_{t} \in[\tau, n \bar{e}+\tau)$ and $e_{i t}=\frac{w_{t}}{n}$ if $w_{t}<\tau$. Choose $e_{i L}=\min \left\{\bar{e}, \frac{w_{L}}{n}\right\}$ if $w_{L}<n \bar{e}+\tau$.

Proof. See Appendix A.

Proposition 2 gives us the following results:

Result 1. If $\bar{w}-\frac{1-\beta}{\beta} n \bar{e} \geq n \bar{e}+\tau$ then the strategy stated in proposition 2 is a SPNE if used by all players. Moreover, if $w_{1} \geq n \bar{e}+\tau$ then on the equilibrium path all players will exert effort $\bar{e}$ in all periods. If $w_{1} \in[\tau, n \bar{e}+\tau)$ players will exert effort $\bar{e}$ in all periods but the first, where they will put effort $\frac{w_{1}-\tau}{n}$. If $w_{1}<\tau$ then in period 1 all players will exert effort $\frac{w_{1}}{n}$ and 0 effort in the remaining periods.

Result 2. If $\bar{w}-\frac{1-\beta}{\beta} n \bar{e}<n \bar{e}+\tau$ then the strategy stated in proposition 2 is a SPNE if used by all players. Moreover, if $w_{1} \in[\tau, n \bar{e}+\tau)$ players will exert effort $\frac{w_{1}-\tau}{n}$ in period 1; efforts $\frac{\beta(\bar{w}-\tau)}{n}$ in periods 2 to $L-1$ and effort $\min \left\{\bar{e}, \frac{\tau+\beta(\bar{w}-\tau)}{n}\right\}$ in period $L$. If $w_{1} \geq n \bar{e}+\tau$ and $L<M$ then on the equilibrium path all players will exert effort $\bar{e}$ in all periods. ${ }^{5}$ If $w_{1} \geq n \bar{e}+\tau$ and $L \geq M$ then on the equilibrium path all players will exert effort $\bar{e}$ in periods 1 to $M-1$ and efforts like in case $w_{1} \in[\tau, n \bar{e}+\tau)$ afterwards.

\footnotetext{
${ }^{5}$ See Lemma 1 for the value of $M$.
} 
If $w_{1}<\tau$ then in period 1 all players will exert effort $\frac{w_{1}}{n}$ and 0 effort in the remaining periods.

The SPNE constructed above holds for relatively low values of $\tau(\tau \leq \bar{e})$ and/or relatively high levels of $\beta\left(\beta \geq \frac{\tau(n-1)}{\bar{w}-\tau}\right)$ because in those cases, the temptation to exert high effort so that the resource is depleted below $\tau$, is overcome by the high resource growth resulting from exerting less effort today. However, if $\beta$ is low, then this trade-off disappears and the only SPNE is for all players to exert the highest possible symmetric effort. The following proposition describes the result.

Proposition 3. If $\beta \leq \frac{\tau(n-1)}{\bar{w}-\tau}$ and $\tau \leq \bar{e}$ then the following strategy used by all players constitutes a SPNE. In all periods $t=1 . .2$ choose $e_{i t}=\min \left\{\bar{e}, \frac{w_{t}}{n}\right\}$.

Proof. See Appendix A.

Figure 1 shows equilibrium paths for different initial conditions and different parameter combinations (as in the two propositions above). It can be easily seen that for the assumptions of proposition 2 the resource stock converges to the level $\tau+\beta(\bar{w}-\tau)$ and for the assumptions of proposition 3 the resource stock converges to 0 .

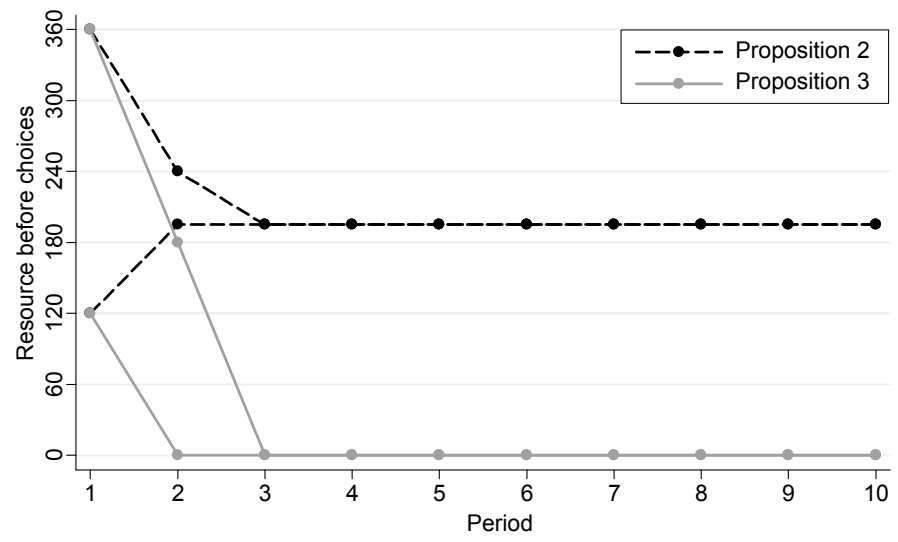

Figure 1: Possible equilibrium paths for parameter assumptions in propositions 2 and 3 . We display the paths for two different initial resource stocks $w_{1}: 360$ and 120 . The growth rates $(\beta)$ depicted here are exactly those from the experimental CPRL $(0.25)$ and CPRH (0.50) treatments (see Section 3).

An important observation about propositions 2 and 3 should be made. In the interesting case $\bar{w}-\frac{1-\beta}{\beta} n \bar{e}<n \bar{e}$, where even with high $w_{1}$, exerting efforts $\bar{e}$ eventually depletes the resource to a level less than $n \bar{e}$ (so that resource can be depleted), for any given parameters only one of the two SPNE described in the propositions above can 
occur. $^{6}$ This is clear from the proofs of Case 2.1 of proposition 2 and the first case of proposition 3: the restrictions on $\beta$ ( $\beta \geq \frac{\tau(n-1)}{\bar{w}-\tau}$ and $\beta \leq \frac{\tau(n-1)}{\bar{w}-\tau}$ correspondingly) are equivalent to the no profitable deviation conditions. Therefore, our model predicts a unique symmetric Markov SPNE for any set of parameters. ${ }^{7}$

Result 3. If $\beta \leq \frac{\tau(n-1)}{\bar{w}-\tau}$ and $\tau \leq \bar{e}$ then the strategy stated in proposition 3 is a SPNE if played by all players. On the equilibrium path players harvest $\bar{e}$ if the resource is higher than $n \bar{e}$ and harvest $1 / n$ fraction of the resource otherwise.

Thus, while in some cases, our model predicts overharvesting of CPRs in line with the classic statement of the commons problem, we also find that in equilibrium for a range of parameters, there is no commons dilemma. For a sufficient growth rate, CPRs can be sustained indefinitely.

\subsection{Social Planner's Problem}

In this section we consider the same problem from the perspective of a Social Planner who seeks to maximize the sum of individual utilities in our CPR game. Without loss of generality we will solve for the optimal harvesting strategy in the single player case. In each period $t$, the social planner's payoff is defined by the first part of equation (1):

$$
\pi\left(e_{t} \mid w_{t}\right)=\bar{e}+(\alpha-1) e_{t} \text { where } 0 \leq e_{t} \leq \min \left\{\bar{e}, w_{t}\right\} .
$$

In what follows we will again abuse notation and assume that $\pi\left(e_{t} \mid w_{t}\right)=e_{t}$ without loss of generality.

Given the initial resource stock $w_{1}$, a Social Planner faces the following problem:

$$
\begin{array}{ll} 
& \max _{\left(e_{t}\right)_{t=1 . L}} \sum_{t=1}^{L} e_{t} \\
\text { s.t. } & w_{t+1}=w_{t}-e_{t}+\beta\left(\bar{w}-\left(w_{t}-e_{t}\right)\right) \mathbb{1}_{w_{t}-e_{t} \geq \tau} \\
& 0 \leq e_{t} \leq \min \left\{\bar{e}, w_{t}\right\} .
\end{array}
$$

Before proceeding to the Proposition describing the solution to this problem, let us first make a useful observation. By Lemma 2 no solution to the above problem can have $w_{t}<\tau$ for $t=1 . . L-1$. Thus, we can simplify the problem by removing the

\footnotetext{
${ }^{6}$ Unless $\beta=\frac{\tau(n-1)}{\bar{w}-\tau}$ so that players are indifferent between both SPNE.

${ }^{7}$ Apart from the case $\beta=\frac{\tau(n-1)}{\bar{w}-\tau}$ when two equilibria exist.
} 
(never optimal) choices that lead to the case $w_{t}<\tau$ :

$$
\begin{array}{ll} 
& \max _{\left(e_{t}\right)_{t=1 . L}} \sum_{t=1}^{L} e_{t} \\
\text { s.t. } & w_{t+1}=w_{t}-e_{t}+\beta\left(\bar{w}-\left(w_{t}-e_{t}\right)\right)=(1-\beta)\left(w_{t}-e_{t}\right)+\beta \bar{w} \\
& 0 \leq e_{t} \leq \min \left\{\bar{e}, w_{t}-\tau\right\} \text { for } t=1 . . L-1 \\
& 0 \leq e_{L} \leq \min \left\{\bar{e}, w_{L}\right\} .
\end{array}
$$

The solution to problem (3) must be the same as to problem (2).

Now we are ready to find the solution.

Proposition 4. The following choice procedure generates the solution to the Social Planner's problem (2) as long as $\tau<\bar{e}$. In periods $t=1 . . L, e_{t}=\bar{e}$ if the resource before period $t$ is $w_{t} \geq \bar{e}+\tau$. However, in period $t=1 . . L-1, e_{t}=w_{t}-\tau$ if $w_{t} \in[\tau, \bar{e}+\tau)$ and $e_{t}=w_{t}$ if $w_{t}<\tau$. If $w_{L}<\bar{e}+\tau$ then $e_{L}=\min \left\{\bar{e}, w_{L}\right\}$.

Proof. See Appendix A.

Note that the Social Planner's optimal plan precisely mimics the strategies of players in the SPNE of the game with a high resource regrowth rate as described in Proposition 2 for all combinations of parameters. However, with a low regrowth rate, the planner would prefer to sustain the resource while individuals rapidly deplete it in equilibrium. ${ }^{8}$ Thus, for low growth rate CPR systems, this finding highlights the tension at the heart of the commons problem and provides an additional benchmark against which to compare behavior.

\subsection{Comparison to Other CPR Models}

Our model differs from the classical common pool resource games reported in previous literature (Gardner, Ostrom, and Walker, 1990; Walker, Gardner, and Ostrom, 1990; Walker and Gardner, 1992; Ostrom, Walker, and Gardner, 1994; Falk, Fehr, and

\footnotetext{
${ }^{8}$ One caveat is that, for the planner's solution to correspond in this way, we must assume the absence of externalities associated with extraction of the resource. If, for example, some members of society are not involved in resource extraction but value the resource stock for other reasons, then the Social Planner's solution will not correspond to the SPNE of the high-growth game. Suppose that the resource is a forest in which some members of society enjoy hiking. In Appendix $C$ we find the solution to the Social Planner's problem with a negative externality of extraction. We assume that there is some level of the resource $\eta>\tau$ such that if the resource is above that level then some additional utility $u$ is enjoyed by the society. Proposition 5 in Appendix $C$ shows that in this case the optimal solution is to deplete the resource to some level above $\tau$ so that the resource grows between periods to exactly $\eta$. It is important to notice that this is true for all possible growth rates of the resource.
} 
Fischbacher, 2002; Velez, Stranlund, and Murphy, 2009). In the classical repeated CPR game, the stage game payoff is defined by $\pi_{i}=e-x_{i}+\left(x_{i} / \sum x_{i}\right) F\left(\sum x_{i}\right)$, where $F$ is a concave function and $x_{i}$ is the amount extracted by player $i$. This models a situation in which each person's resource extraction imposes a direct negative externality on others. With this payoff function there is a Nash equilibrium of the stage game where each player extracts some amount of the CPR, but it is not depleted (Walker and Gardner, 1992). We adopt a more extreme version of payoffs so that the unique $\mathrm{NE}$ of the stage game is to deplete the resource.

The most important difference between the classical model and ours is how the game is repeated, and this feature explains why, despite the extreme predictions for our stage game, we find that the CPR can be sustained in some equilbria. In the classical setup, the stage game in each period is the same. We think that this modeling decision is rather restrictive, because this makes it impossible to study the effects of resource growth from period to period on the equilibrium decisions. In our model resource stocks replenish over time, so long as they are maintained above a certain minimum. Therefore, our CPR game is a repeated game with a state variable that more nearly captures features of naturally occurring CPRs such as fisheries, where resource stocks are path dependent. Bru, Cabrera, Capra, and Gomez (2003) also consider a CPR game where the resource stock in each period depends on the extraction decisions in previous periods. However, their setup is also more restrictive: 2 players move sequentially and can only choose between high or low extraction rates. In our model the decisions of $n$ players in each period are simultaneous, which may be more realistic for certain types of $\mathrm{CPR},{ }^{9}$ and the amount extracted can be any real number. It should be noted though, that qualitatively the model in Bru, Cabrera, Capra, and Gomez (2003) has similar predictions to our model: in equilibrium the resource is either depleted or is sustained depending on ecological parameters.

\section{Experimental Design}

As noted above, we test the predictions of our model in the lab, and we also explore the impact of group composition on CPR sustainability. The experiment consists of two decision-making stages and a questionnaire.

\footnotetext{
${ }^{9}$ For example, when fishermen decide how much fish to catch they do not know the decisions of other fishermen who are at sea at the same time.
} 


\subsection{Rule Following Task}

In stage 1, which we call the Rule Following stage (RF), subjects control a stick figure walking across the computer screen (this task is drawn from Kimbrough and Vostroknutov, 2012). Each subject makes 5 decisions concerning the amount of time they wait at a sequence of red traffic lights, each of which will turn green 5 seconds after their arrival. Figure 2 shows the screen that the subjects see.

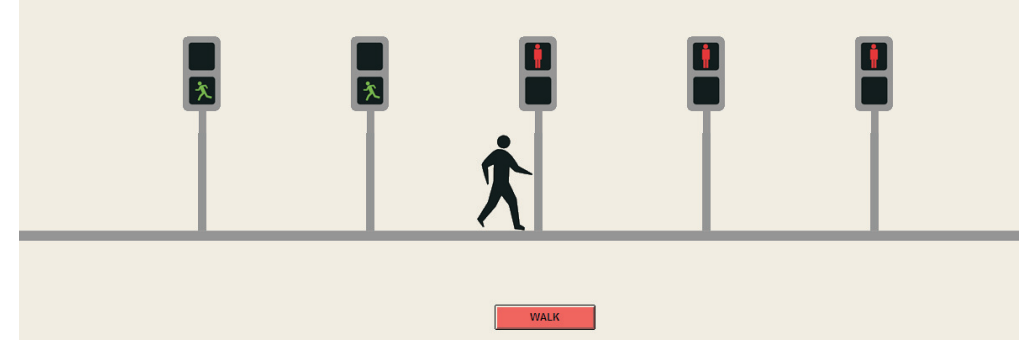

Figure 2: Screen shot of the Rule Following stage.

The following description of the RF stage closely follows that in Kimbrough and Vostroknutov (2012). At the beginning of the RF stage, the stick figure is standing at the left border of the screen, and all traffic lights are red. ${ }^{10}$ Subjects initiate the RF stage by pressing the START button. At this moment, the stick figure starts walking towards the first traffic light. Upon reaching the first red light, the stick figure automatically stops. The light turns green 5 seconds after the stick figure stops; however, subjects are free to press a button labeled WALK any time after the stick figure stops. When a subject presses WALK, the stick figure continues walking to the next red light before stopping again, and subjects must once again press WALK to continue to the next light. Throughout the RF stage, the WALK button is shown in the middle of the screen. Subjects can press the WALK button at any time during the RF stage. However, it becomes functional only when the stick figure stops at a traffic light.

Subjects receive an endowment of 8 Euro, and they are told that for each second they spend in the RF stage they will lose 0.08 Euro. It takes 4 seconds to walk between each traffic light, and 4 seconds from the final light to the finish. Therefore, all subjects lose around 2 Euro walking, and if a subject waits for green at all 5 traffic lights, she will lose an additional 2 Euro waiting. Thus the most a subject can earn in the RF stage is 6 Euro (if she spends no time waiting at traffic lights), and the most she can earn if she waits is 4 Euro (if she waits exactly 5 seconds at each light). In the instructions

\footnotetext{
${ }^{10}$ Before starting the task, subjects see a short cartoon in which the traffic lights blink from red to green to ensure they understand that the lights can turn green.
} 
for the RF stage (see Appendix F) subjects are told: "The rule is to wait at each stop light until it turns green". No other information, apart from the payment scheme and a general description of the walking procedure, is provided in the instructions. ${ }^{11}$

The rule following task instructs subjects to follow a familiar, arbitrary rule at some cost to themselves. Obedience to this rule is in some ways "irrational" in the sense that with no monitoring or penalties, there is no cost to breaking the rule. In our experiment the typical justification for obeying traffic law is irrelevant because there are no other drivers or pedestrians to protect or be protected from - this is similar to a situation in which people wait at stoplights in the middle of the night, despite the absence of other pedestrians or drivers. Why individuals are willing to incur costs in service of a rule is an open question. More important for our purposes, we argue that the decision to follow costly rules reveals information about an individual's behavioral type: those who incur costs in order to follow rules implicitly identify themselves as conditional cooperators or "team players".

We test this hypothesis and the hypotheses derived from our model in two treatments: the CPR game with high $\beta(\mathrm{CPRH})$ and the CPR game with low $\beta$ (CPRL). Stage 1 of both treatments is the Rule Following task as described above. In stage 2 of both treatments subjects play 10 periods of a repeated CPR game in fixed groups of 4 .

\subsection{CPR Experiment}

Before making decisions in the RF stage, subjects only receive instructions for that stage. In particular, they are aware that the experiment will consist of several stages, but they know neither what they will do in the next stage(s) nor the connection between the RF stage and consecutive stages. ${ }^{12}$ Unknown to the subjects, their decisions

\footnotetext{
${ }^{11}$ If a subject asked what would happen if he/she passed through the red light, an experimenter explained that all information relevant to the experiment is presented in the instructions.

${ }^{12}$ Specifically, subjects' rule-following task instructions read "Part I" at the top of the page (see Appendix F). Some might be concerned that the foreknowledge of a second task will influence behavior in the first task. However, as we noted in a prevous implementation of a similar experimental design in Kimbrough and Vostroknutov (2012):
}

In dictator game experiments, knowledge of the existence of an unspecified second-stage has been shown to alter subjects' behavior by making them more cooperative in expectation that their first-stage behavior may influence their second-stage reputation (Smith, 2008). If subjects are concerned for their reputation and thus wait longer than they might in a treatment without an implicit "shadow of the future" (or, similarly, with a double-blind protocol), this would dilute the information content of the rule-following task, thereby strengthening our results. 
in the RF stage determine their group membership in the CPR game.

We employ the same matching procedure in both treatments. First, we randomly divide subjects into groups of 8 . Second, within each group of 8 , we rank subjects according to the total time they spent waiting at traffic lights-at least 25 seconds for those subjects who waited for green at all traffic lights and close to 0 seconds for those who did not wait at any traffic light. Then, in each group of 8 , we separate the top 4 subjects (Rule-Followers) and the bottom 4 subjects (Rule-Breakers) into two groups for stage 2. After we match subjects, there is no interaction between any groups of 4 . Subjects are not informed about the matching procedure, and they are told only that they will now interact with a fixed group of three other participants (see Appendix $\mathrm{G}){ }^{13}$

In the CPRL treatment the CPR game is played in each group of 4 subjects for 10 periods. ${ }^{14}$ Subjects are told that in each period they will collect 60 tokens from a group account and a private account. Their decision is how many of these 60 tokens to take from each account. Each token taken from the private account yields a return of 1 Euro cent per token. Whereas, each token taken from the group account generates a return of 2 Euro cents. Token supply in the private account is unlimited, which means that each subject can take as many tokens as she wishes from the private account in each period (subject to the 60 token limit). However, the group account, from which all four subjects can take tokens, has a limited capacity and initially contains a total of 360 tokens. Whenever subjects take tokens from the group account, its size diminishes by the sum of tokens taken. Before the next period, the group account replenishes: if there are $X$ tokens remaining, then next period the group account will contain $X+\beta(360-X)$ tokens (here $\beta$ is treatment dependent growth rate). However, if the number of tokens remaining falls under 30 tokens, then the group account won't replenish. Finally, if subjects choose to take more tokens than there are in the group account, tokens are divided according to the amounts subjects have chosen. ${ }^{15}$ Thus, in CPRH treatment the parameters are as follows: $\bar{w}=360, \bar{e}=60, n=4 ; \beta=0.5$, $\tau=30, \alpha=2, L=10, M=2$. Notice that with these parameters we have

$$
\beta>\frac{\tau(n-1)}{\bar{w}-\tau}
$$

\footnotetext{
${ }^{13}$ This is not deception since none of the statements in the instructions are false or misleading.

${ }^{14}$ Subjects are not informed how many periods of the CPR task they will perform. The instructions in Appendix G specify that the game will be played for several periods. An indefinite ending point should provide a best-case scenario for cooperation, regardless of treatment.

${ }^{15}$ See Appendix $\mathrm{G}$ for the exact rule of how tokens are divided among subjects in this case.
} 
which means that the SPNE is to preserve the resource indefinitly (see Proposition 2). The CPRL treatment is the same as CPRH except one change. Here $\beta=0.25$ and thus is lower than in the CPRH treatment. This switches the sign of the inequality above so that the SPNE is as depicted in Proposition 3 and the resource will be rapidly depleted in equilibrium. To isolate the impact of varying $\beta$ in the absence of sorting we ran two additional treatments where the order of the tasks was reversed and subjects were placed into groups at random (revCPRL and revCPRH). In appendix E we also report an exponential growth treatment, where a different resource regeneration function was used (CPREXP). Finally, at the end of the experiment, subjects filled out the Moral Foundations Questionnaire (Haidt, 2007), which provides estimates of subjects concerns for 5 fundamental moral issues.

Overall, 88 subjects participated in the CPRH treatment; 96 subjects in the CPRL treatment; 24 subjects in revCPRH; 32 subjects in revCPRL; and 56 in CPREXP (reported in Appendix E). No pilots or other treatments were run. All experiments were conducted at the Behavioral and Experimental Economics Laboratory (BEELab) at Maastricht University in October-November 2011. Experiments were programmed in $z$-Tree (Fischbacher, 2007).

\subsection{Related Experiments}

The pioneering CPR experiments are Gardner, Ostrom, and Walker (1990), Walker, Gardner, and Ostrom (1990), and Walker and Gardner (1992). Here 1) the stage game has a NE which is close to social optimum and in which the CPR is not depleted, and 2) there is no path-dependence of resource stocks on past choices. The main finding is excess depletion of the resource. In our experiment the stage game has a unique NE in which the resource is totally depleted; thus resource stocks may be very difficult to sustain. More recent experiments with CPR games follow two main paths: 1) asymmetric CPR games which mimic specific problems found in the field (Cárdenas, Janssen, and Bousquet, 2011; Janssen, Anderies, and Joshi, 2011); 2) studies that investigate how different institutional arrangements influence CPR extraction (Cárdenas and Ostrom, 2004; Cárdenas, Janssen, and Bousquet, 2011; RodriguezSickert, Guzmán, and Cárdenas, 2008); 3) studies that compare behavior to models of various preference structures (Velez, Stranlund, and Murphy, 2009). All these studies use the classical CPR game as introduced by Walker and Gardner (1992), which by the static nature of the game does not allow them to answer questions related to resource regrowth. Neither does any of them employ assortative matching. 
The experiment most similar to ours is the Forestry Game reported in Cárdenas, Janssen, and Bousquet (2011). Analogously to our experiment the resource grows after each period. The growth is very slow and the resource is quickly depleted in all groups. This is in line with our results: participants cannot sustain the resource with low growth rates. Another experiment with resource regrowth is conducted by Bru, Cabrera, Capra, and Gomez (2003). Their game is rather different from ours (players move sequentially), but the authors also find overharvesting. A somewhat different setup is used in Janssen, Holahan, Lee, and Ostrom (2010), where participants control virtual avatars as they travel around an on-screen matrix and collect tokens, which regrow with time. Overharvesting is immanent, however it is mitigated by communication and punishment. Janssen (2010) reports additional experiments using the same experimental environment and exploring the effects of communication and punishment on the endogenous creation of informal CPR management institutions under different resource growth rates, and he reports that groups tend to preserve the resource successfully whether growth is high or low, in line with previous experiments on the effects of communication and punishment on CPR management.

\section{Experimental Findings}

First, we compare the behavior of rule-breaking and rule-following groups in the CPRH and CPRL treatments to the SPNE predictions and to one another. Then we explore how individual differences in the willingness to follow costly rules drive individual behavior in the CPR game. Then, we examine behavior in our reverse treatments to isolate the separate effects of ecology and the sorting mechanism on resource extraction decisions.

\subsection{CPRH Treatment}

At the beginning of the $\mathrm{CPRH}$ treatment, subjects are divided into rule-breaking and rule-following groups as described above. Average waiting times in rule-breaking and rule-following groups are 14.76 and 27.79 seconds respectively. ${ }^{16}$ We first look at the evolution of the resource stock in each group separately. Figure 3 displays time series of resource stocks in each group of the CPRH treatment. The top row shows rule-breaking groups, and the bottom row shows rule-following groups. Here, the SPNE of the repeated game with CPRH parameters $(\beta=0.5)$ predicts that all groups

\footnotetext{
${ }^{16}$ See Figure E1 in Appendix E for histograms of waiting times by type and treatment.
} 
will sustain the resource for the full 10 periods, but the majority of groups exhaust the resource rather early.

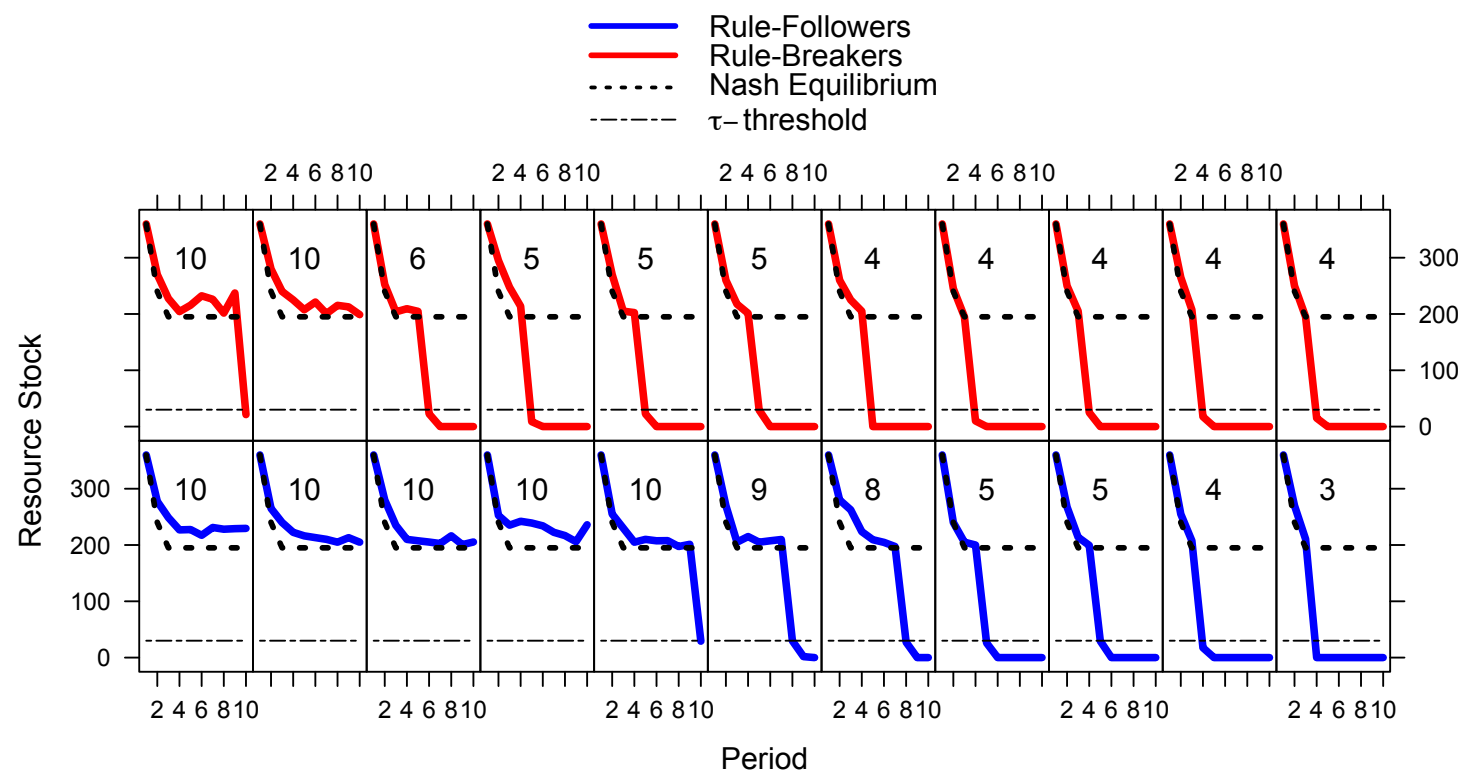

Figure 3: Time series of resource stocks for individual groups in CPRH treatment. The numbers in the middle of each panel indicate the period in which the group exhausted the resource.

The numbers in the center of each panel specify the period in which the group exhausted the resource. We denote this variable exhaustion. ${ }^{17}$ Among rule-followers, 4 out of 11 groups (36\%) sustain the resource at or above the equilibrium level for all 10 periods, while among rule-breakers there is only 1 such group (9\%). More generally, $45 \%$ of rule-following groups sustain some amount of the resource for all 10 periods while only $18 \%$ of rule-breaking groups did so. The rest of the groups deviate from the SPNE prediction and harvest the resource until it is eventually depleted. Nevertheless, a permutation test indicates that there is a significant difference in the mean period of exhaustion between rule-followers and rule-breakers in the CPRH treatment (means are 7.64 and 5.55, respectively; $p$-value $=0.041$, one-sided test).

\subsection{Cooperation and Coordination in the CPRH Treatment}

In part the relatively low success rate in sustaining the resource can be attributed to the complexity of the coordination problem in equilibrium. To sustain the resource beyond period 2, 4 individuals must each expend effort less or equal to $\frac{w_{t}-\tau}{4}$. Since

\footnotetext{
${ }^{17}$ If the group never exhausts the resource, exhaustion is 10.
} 
all four seek to maximize their effort, there is a substantial probability that the resource capacity will fall below $\tau$ and the resource will be depleted, if even one subject harvests a little bit more than $\frac{w_{t}-\tau}{4} \cdot{ }^{18}$ From Figure 3 it is clear that many groups of both rule-followers and rule-breakers failed to solve this coordination problem. Specifically, note the number of cases in which the resource stock falls from around the equilibrium level at time $t$ into the interval $(0, \tau)$ at time $t+1$. In all such cases, $w_{t}>\sum_{i \in I} e_{i}>w_{t}-\tau$, which indicates that at least some subjects chose their effort levels with the $\tau$ threshold in mind.

Moreover, if we compare rule-following to rule-breaking groups, restricting attention only to those groups that depleted the resource below $\tau$, we find that rulebreakers tend to overshoot the $\tau$-threshold by a significantly larger margin than rulefollowers. Let $w_{j, k}$ be the resource stock in group $j$ at the beginning of the period $k$, which is the period after which the total stock first fell below the $\tau$-threshold. Then $x_{j}=\tau-w_{j, k}$ is the amount by which a group overshot $\tau$. The median value of $x_{j}$ is 2.7 among rule-followers and 10.6 among rule-breakers, and a Wilcoxon rank-sum test rejects the null hypothesis that $\bar{x}_{\text {followers }}=\bar{x}_{\text {breakers }}$ in favor of the alternative hypothesis that $\bar{x}_{\text {followers }}<\bar{x}_{\text {breakers }}\left(W_{7,10}=18, p\right.$-value $=0.053$, one-sided test, means are 7.3 and 12.8 , respectively).

This provides evidence that resource depletion among rule-breakers has a different source than among rule-followers. Specifically, rule-following groups often deplete the resource due to the difficulty of the coordination problem in equilibrium and not necessarily because many individuals extract more than their $\frac{w_{t}-\tau}{4}$ share of the resource. On the other hand, rule-breaking groups tend to substantially overharvest the resource, suggesting that they are less likely to try to preserve the resource in the first place; that is, they do not even attempt to solve the coordination problem. As additional evidence, Spearman's test of rank correlation between $\bar{x}$ and exhaustion is negative and highly significant $(\rho=-0.66$. $p$-value $=0.003)$. In Section 4.3 below, we also show that individual differences in rule-following correlate strongly with individual efforts to preserve the resource in all treatments.

\subsection{CPRL Treatment}

Now we compare behavior of rule-following and rule-breaking groups in the CPRL treatment. Average waiting times in rule-breaking and rule-following groups are sim-

\footnotetext{
${ }^{18}$ Moreover, given that subjects' effort choices were integer constrained, when $\left(\frac{w_{t}-\tau}{4} \bmod 4\right) \neq 0$, the coordination problem is even more complicated.
} 
ilar to those observed in the CPRH treatment at 15.22 and 30.38 seconds, respectively. However, in the CPRL treatment, all groups eventually exhaust the resource, which is qualitatively consistent with the SPNE of the repeated game with CPRL parameters. Strictly speaking, only 3 groups play the SPNE and exhaust the resource in 2 periods. Instead, the majority of groups manage to sustain resource stocks at positive levels for 3 or more periods, and many of the groups that deplete the resource exhibit behavior similar to that described in the CPRH treatment wherein the stock falls to just below $\tau$ before being completely depleted in the following period. This indicates that subjects understand the trade-off between consuming as much of the resource as possible today and withholding harvesting today to sustain yields over many periods, and it suggests that some subjects seek to improve total social welfare by restraining their harvest even in the CPRL treatment. We will return to this point later.

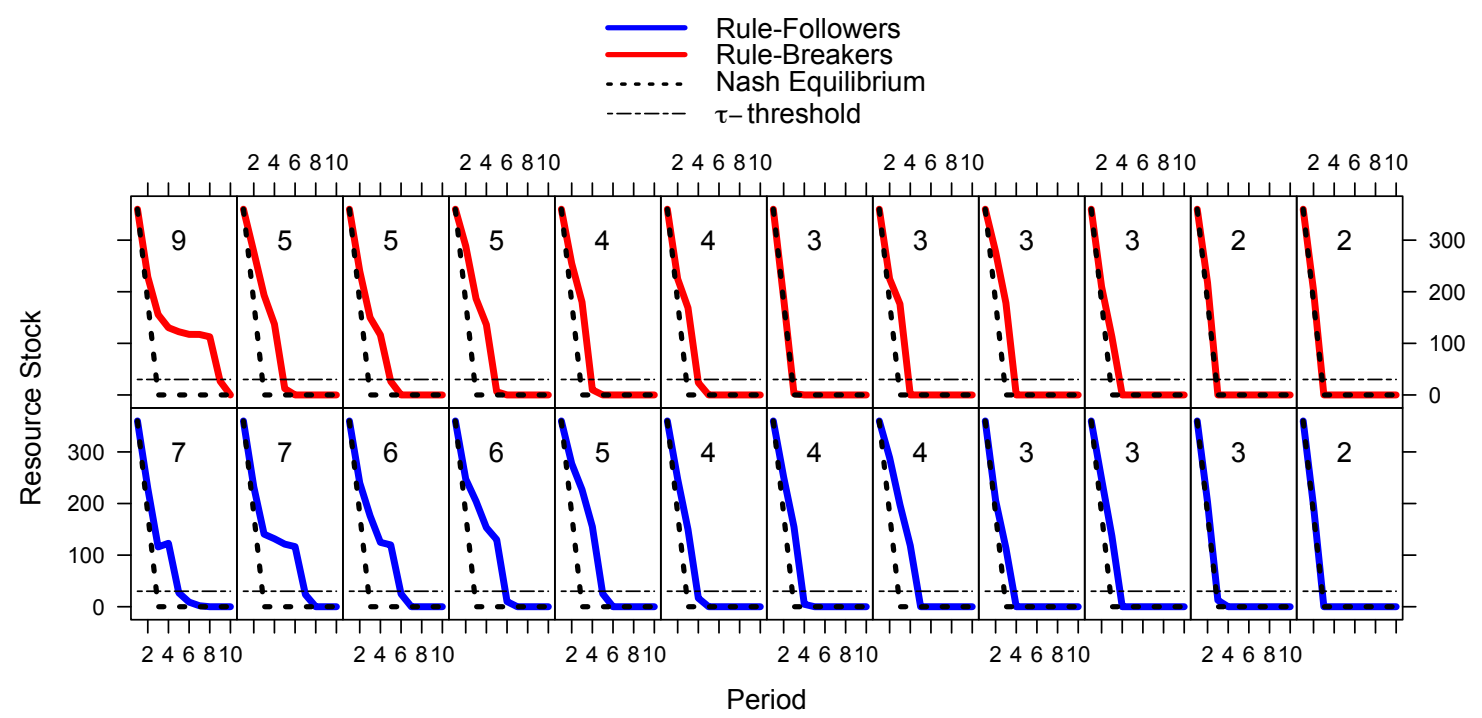

Figure 4: Time series of resource stocks by group in the CPRL treatment. The numbers in the middle of each panel indicate the period in which the group exhausted the resource stock (exhaustion).

A permutation test indicates that there is no significant difference in the mean period of exhaustion between rule-followers and rule-breakers in the CPRL treatment (means are 4.5 and 4 , respectively; $p$-value $=0.29$, one-sided test). Here, given the slow rate of resource regeneration $(\beta=0.25)$, subjects are unable to permanently sustain the resource above the $\tau$ threshold, regardless of their type. ${ }^{19}$

In order to confirm that the change in $\beta$ between treatments impacts behavior, we

\footnotetext{
${ }^{19}$ This is consistent with recent evidence suggesting that levels of cooperation across groups within the same society are sensitive to ecological variables (Lamba and Mace, 2011).
} 
compare the distribution of exhaustion for rule-breakers and rule-followers across treatments as well as the overall treatment difference. Permutation tests demonstrate that all three comparisons are significant indicating that the mean period of exhaustion is later in the CPRH treatment, consistent with the comparative statics predictions of the model. Rule-breakers in CPRL versus CPRH treatment: $p$-value $=0.060$, one-sided test; Rule-followers: $p$-value $=0.006$, one-sided test; all groups by treatment: $p$-value $=0.001$, one-sided test. ${ }^{20}$

Thus we find compelling evidence that behavior in the CPR game is sensitive to ecological characteristics of the resource system and to the behavioral type-composition of groups. The following three findings summarize our main results on the separate and joint role of social and ecological variables on successful CPR management:

Finding 1. In the CPRH treatment half of rule-following groups sustain the resource as the SPNE predicts. However, contrary to the SPNE prediction the remaining rulefollowing groups and almost all rule-breaking groups deplete the resource. There is a significant difference between rule-breakers and rule-followers.

Finding 2. In the CPRL treatment all groups eventually exhaust the resource, though it takes them longer than 2 periods and thus behavior deviates from the SPNE. There is no significant difference between rule-breakers and rule-followers.

Finding 3. As predicted by the model, differences in the resource regeneration rate, $\beta$, lead to significant differences between the CPRL and CPRH treatments. Comparing rule-breakers and rule-followers across treatments and comparing all groups in each treatment, the mean period of exhaustion is later in the CPRH treatment.

\subsection{Reverse Treatments}

In order to disentangle the effects of the our social and ecological treatment variables, we examine group dynamics in mixed-type groups composed of both rule-breakers and rule-followers. Thus we describe results from two additional control treatments in which we reverse the order of the tasks. We call these reverse treatments, revCPRH and revCPRL. These experiments are identical to the main treatments, except that

\footnotetext{
${ }^{20}$ Further evidence that low growth rates impede sustainability of the resource is provided by our CPREXP treatment in which growth rate was exponential (reported in Appendix E.2). An exponential regrowth function implies that growth is low for low resource stocks and high for high stocks, which is opposite to the CPRL and CPRH treatments. Subjects in all but one group of the CPREXP treatment (both rule-breaking and rule-following groups) exhaust the resource rather quickly, which is similar to the CPRL treatment.
} 
subjects first play CPR game in randomly matched groups of 4 , and then they participate in the RF task. These treatments further allow us to distinguish the effects of individual differences and our sorting mechanism based on the rule-following task. Figures 5 and 6 show group dynamics in the revCRPH and revCPRL treatment.

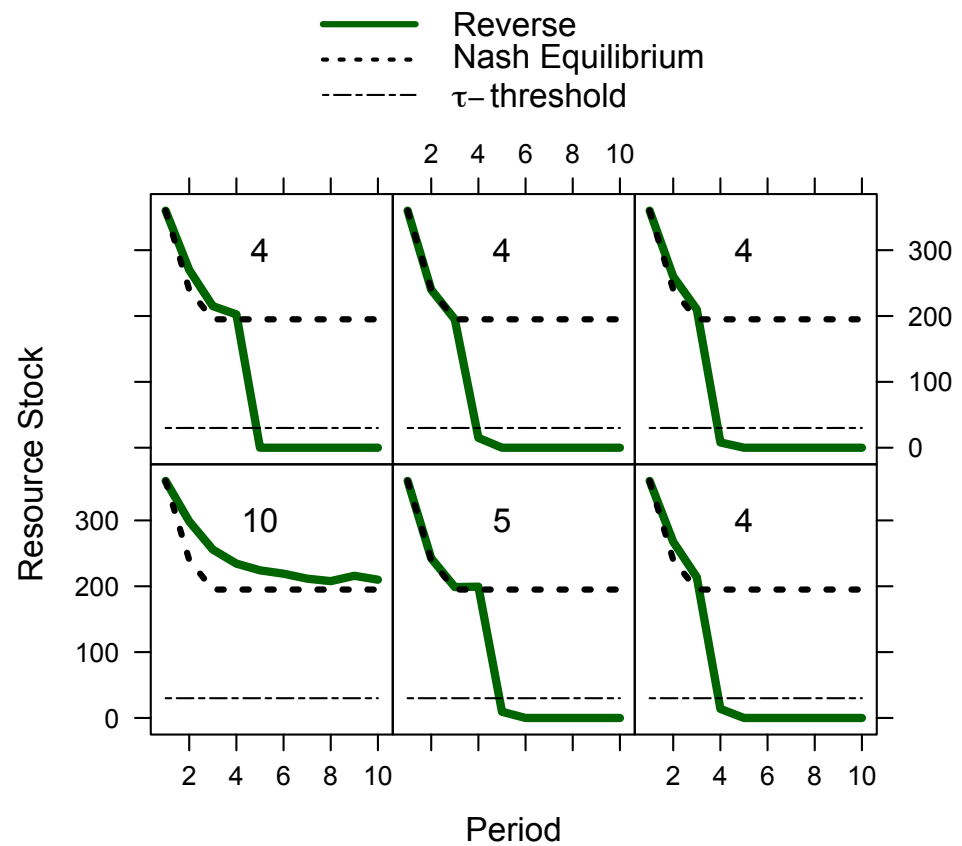

Figure 5: Time series of resource stocks in the revCPRH treatment.

First note that the dynamics in the 6 groups of the revCPRH treatment are qualitatively similar to those observed in the main CPRH treatment in that most groups do not immediately deplete the resource, and one sustains it for the entire 10 periods. However, we can weakly reject the hypothesis of equal mean periods of exhaustion between rule-following groups in the CPRH treatment and the revCPRH treatment (means are 7.64 and 5.17, respectively, one-sided permutation test, $p$-value $=0.066$ ). The same test fails if we compare rule-breaking groups in the CPRH treatment (mean 5.55 ) with revCPRH ( $p$-value $=0.395$ ). This indicates that the presence of rulebreakers offsets the beneficial impact of rule-followers so much that mixed-type groups are indistinguishable from rule-breaking groups. However, through screening and assortative matching, groups of rule-followers can substantially improve CPR management.

Observed behavior in the revCPRL treatment is roughly the same as in the main CPRL treatment, and permutation tests cannot reject the null hypothesis of equal mean exhaustion when comparing revCPRL to either rule-followers or rule-breakers 


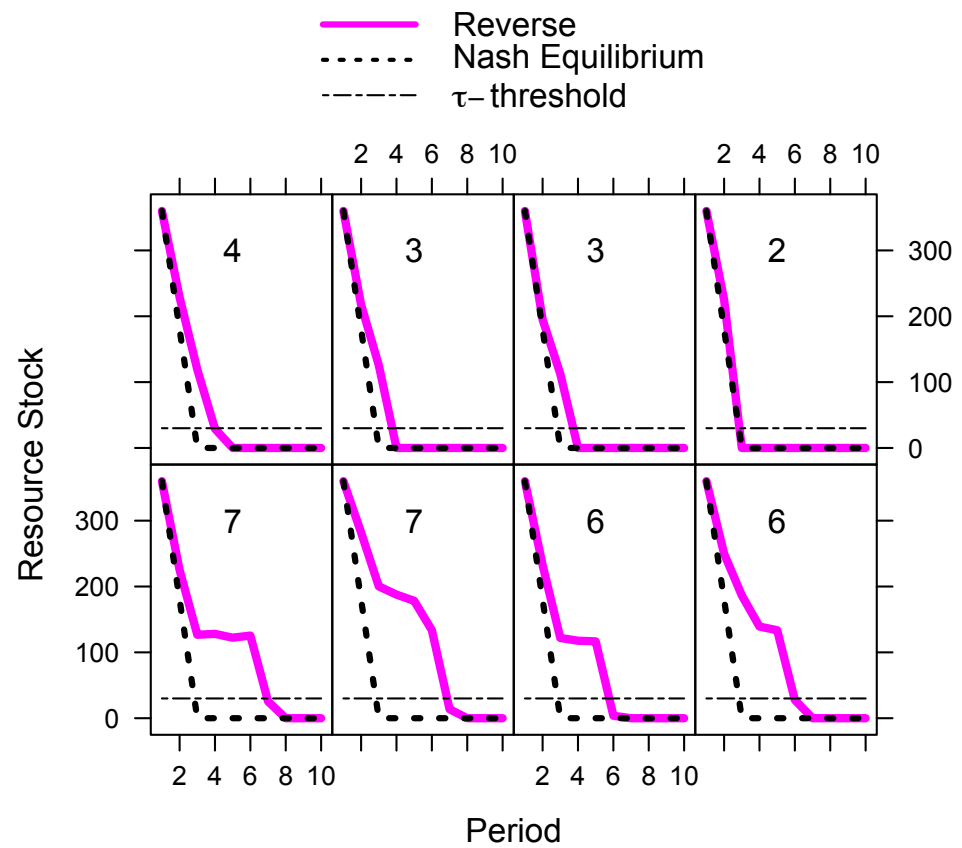

Figure 6: Time series of resource stocks in the revCPRL treatment.

in the CPRL treatment ( $p$-values $=0.801$ and 0.422 , respectively, two-sided tests). Again, the reason for this is the low regeneration rate. Though, we still see that all but one group sustains the resource longer than predicted in the SPNE. This can be attributed to some attempts to preserve the resource, as discussed in the previous section.

Table 1 summarizes the means of exhaustion in all treatments. One can see that mean exhaustion in both revCPRH and revCPRL is lower than in the CPRH treatment and is closer to the CPRL treatment. Since revCPRH and revCPRL treatment groups consist of a mixture of rule-breakers and rule-followers, this analysis supports our hypothesis that only the absence of rule-breakers allows groups to sustain the resource in the CPR game. This finding is similar to that reported in Kimbrough and Vostroknutov (2012) in which play by mixed-type groups is indistinguishable from that of groups composed solely of rule-breakers in both voluntary contribution public goods experiments and trust games, and we provide further evidence that rule-following proclivity predicts conditional cooperation.

Finding 4 . In the reverse treatments group dynamics are indistinguishable from the dynamics of rule-breaking groups in the corresponding main treatments. Rule-following groups in the CPRH treatment sustain the resource longer than revCPRH groups. This 


\begin{tabular}{lcccc}
\hline Mean exhaustion & & & & \\
& CPRH & CPRL & revCPRH & revCPRL \\
\hline Rule-Breakers & 5.55 & 4.00 & & \\
Rule-Followers & 7.64 & 4.50 & & \\
NoSort & & & 5.17 & 4.75 \\
\hline
\end{tabular}

Table 1: Mean values of exhaustion across all treatments.

supports our hypothesis that assortative matching of rule-followers is essential for sustaining the resource.

\subsection{Individual Behavior}

Subjects in the CPR game are driven by two mutually incompatible goals. First, there is the desire to harvest as much of the resource as possible now, in case the resource is exhausted and unavailable in the future; indeed, contrary to the SPNE prediction, in practice this is a concern even in the CPRH treatment. Second, there is a forwardlooking desire to sustain the resource, so it can replenish and be consumed in the future (and this tendency is present even in the CPRL treatment where the SPNE predicts rapid exhaustion). In the CPRH treatment, these motivations are aligned in equilibrium as the solution to the Social Planner's problem corresponds to the SPNE, but in the CPRL treatment the goals are in conflict. ${ }^{21}$ In practice, the path of the resource stock will depend on which of these two goals dominates in the population, and we argue that the goals are associated with individuals of particular types.

In Section 4.2 we showed that rule-following groups in the CPRH treatment often deplete the resource not because they extensively over-harvest the resource, but rather because they often fail to solve the complicated coordination problem in equilibrium. Rule-breaking groups on the other hand, tend to substantially over-harvest the resource. To understand the mechanisms underlying these differences at an individual level we estimate a mixed effects logistic regression in which we analyze time series of individual choices for all subjects in the CPRH, CPRL, revCPRH, and revCPRL treatments. In particular, we are interested in choices that reveal an individual's willingness to show restraint and attempt to preserve the resource. We argue that an individual's choice reveals restraint whenever she chooses to take less than (or equal to) her "share" of the resource stock as defined by the Social Planner's so-

\footnotetext{
${ }^{21}$ In both the CPRL and CPRH treatments the solution to the Social Planner's problem is identical to the SPNE in high- $\beta$ cases like the CPRH treatment (see Section 2.3). Thus, it could be argued that some subjects in the CPRL treatment strive to maximize total social surplus, rather than individual payoffs.
} 
lution to the CPR game, given the current level of the resource (see Section 2.3). We define the dummy variable restraint which is defined for any harvesting effort $(e)$ in any period and any current resource stock $w$. Let $\hat{e}_{w}$ be the optimal effort choice according to the Social Planner's solution to the CPR problem for any given $w$. Then restraint $=1$ if $e \leq \hat{e}_{w}$. In particular, if $w \geq n \bar{e}+\tau=270$ then restraint takes a value of 1 if $e \leq \bar{e}=60 ; 22$ if $w<n \bar{e}+\tau$ then restraint takes a value of 1 as long as $e \leq(w-\tau) / n$; and restraint is 0 otherwise. We exclude observations in which $0 \leq w \leq \tau$ since in such cases the resource has already been depleted. We use a dummy rather than, say, the total deviation from one's "share" because this treats the decision equally for all resource stocks and allows us to normalize interpretation across treatments.

The dependent variable, restraint ${ }_{i, t}$, indicates whether subject $i$ showed restraint in period $t$ and captures the tension between individual and social incentives. In addition to a constant term, our independent variables include: 1) breaker ${ }_{i}$, a dummy indicating whether subject $i$ waited less than 25 seconds in the RF task; 2) CPRH, a dummy for the CPRH treatments; 3 ) Reverse, a dummy for the Reverse treatments and 4 ) others restraint i,t-1 $_{i}$, measuring whether other group members, on aggregate, showed restraint in the previous period. This last variable allows us to estimate the reciprocal response of each subject to the actions of other subjects in their group. We also include an interaction term that allows us to parse the effects of the reciprocity variable more subtly. Specifically, a breaker-others restraint tit-1 $_{i}$ variable allows us to identify whether the types reciprocate in different ways or to different degrees. ${ }^{23}$ Moreover, random effects for each subject-in-group control for repeated measurements at the subject and group level. Table 2 displays the results.

The regression output in column (1) reports the estimated coefficients on our variables of interest, and column (2) reports marginal effects estimated at observed means. First, note that a positive and significant coefficient on the CPRH treatment dummy indicates that subjects respond to higher growth rates with more attempts to preserve the resource. More importantly, a negative and significant coefficient on breaker indicates that rule-breaking individuals are $27 \%$ less likely to show restraint than rule-following individuals. Moreover, both types exhibit significant reciprocal responses (a positive and significant coefficient on others restraint tit-1 $_{i}$ indicates that restraint is met with restraint and lack thereof with lack thereof), an insignificant

\footnotetext{
${ }^{22}$ Note that this condition will always be satisfied; this is true because the Social Planner would always prefer to harvest $n \bar{e}$ when there is no risk of depleting the resource. We cannot simply exclude these observations, though, because our regression employs a lagged variable.

${ }^{23}$ See Appendix D for the definitions of all variables.
} 


\begin{tabular}{lcc}
\hline & $\begin{array}{c}(1) \\
\text { restraint }\end{array}$ & $\begin{array}{c}(2) \\
\text { marginal } \\
\text { effects }\end{array}$ \\
\hline breaker & $-1.234^{* * *}$ & $-0.255^{* * *}$ \\
& $(0.450)$ & $(0.093)$ \\
others restraint ${ }_{t-1}$ & $1.261^{* * *}$ & $0.261^{* * *}$ \\
breaker·others restraint revCPRH $_{t-1}$ & 0.723 & 0.150 \\
& $(0.484)$ & $(0.101)$ \\
CPRH & $1.257^{* * *}$ & $0.260^{* * *}$ \\
& $(0.463)$ & $(0.093)$ \\
Reverse & 0.037 & 0.008 \\
& $(0.518)$ & $(0.107)$ \\
Constant & 0.241 & \\
\hline Log Lik. & $(0.389)$ & \\
\hline N & -456.960 & \\
\hline
\end{tabular}

Standard errors in parentheses.

${ }^{*} p<0.1,{ }^{* *} p<0.05,{ }^{* * *} p<0.01$

Table 2: Mixed effects logistic regression of individual differences in restraint and average marginal effects. Random effects for each group and subject. 856 observations, 240 subjects, 60 independent groups.

interaction between breaker and others restraint $t_{i, t-1}$ would appear to indicate that there are no differences in the reciprocal response between rule-followers and rulebreakers are more strongly reciprocal. However, this coefficient measures both positive and negative reciprocity, and this fact explains why rule-breaking groups tend to deplete the resource more quickly: in this case, rule-breakers are matched with other rule-breakers who are less likely to show restraint in the first place. Reciprocity generates a negative feedback loop whereby rule-breaking groups quickly deplete the resource via negative reciprocal responses to lack of restraint by others. On the other hand, when rule-breakers are matched together with others who are more likely to show restraint, this produces positive feedback, and thus rule-following groups tend to preserve the resource. Thus, differences in the rule-following type-composition of the group explain differences in CPR management dynamics. 
Finding 5. Overall, rule-breakers show less restraint than rule-followers. Both types respond reciprocally to the behavior of others, and as a result, groups that contain rule-breakers rapidly deplete resources due to negative reciprocity.

\subsection{Heterogeneity in Rule-Following}

Unreported regressions replicate the findings of Kimbrough and Vostroknutov (2012) that women are more likely to follow the rule than men and Law students are more likely to break the rule than others. We observe no differences across individuals of different nationalities, but we draw our subjects from a relatively homogeneous pool, mostly composed of western European individuals. We also find no evidence that subjects' moral foundations scores are correlated with rule-following proclivity.

\section{Discussion}

We report experimental tests of a model of the classic common pool resource problem. Our model maintains the features that are typically argued to generate overharvesting of a common pool resource, but we show that depending on the regeneration rate of the resource, individuals may actually preserve the resource indefinitely in equilibrium.

Data from laboratory experiments supports the comparative statics predictions of the model when we compare behavior under two parameter configurations, one in which the resource is sustained in equilibrium and one in which it is exhausted. Nevertheless, individuals destroy the resource more frequently than predicted in the former case and maintain it for longer than predicted in the latter. We show that this behavior also varies with the behavioral-type composition of the group. Specifically, groups composed of rule-breakers are more likely to exhaust the resource than groups of rule-followers, and this is driven by increased impulsivity (lack of restraint) among rule-breakers and by negative reciprocity in response to observed lack of restraint.

While our model helps to account for the diversity of outcomes in common pool resource management around the world, our experimental results show that subtle differences in group composition can also have large impacts. This finding highlights the crucial role of screening, exclusion and assortative matching in the preservation of CPRs, and it reiterates the point that ecological factors are merely permissive and not prescriptive of any observed form of social organization (Algaze, 2005), thereby 
providing additional support for the social-ecological systems view of CPRs.

Successful resolution of social dilemmas requires screening out non-cooperative types, and we argue that one way of identifying those types is to observe their willingness to follow costly rules. When individuals choose to pay costs in order to bind themselves to rules, their actions reveal willingness to cooperate and a far-sighted view of the utility of rules that makes them valuable group members. Crucially, this behavioral tendency is revealed ex ante in the decision not to follow the rule, and thus our daily experience provides copious information about the cooperative tendency of others, even when we are unable to directly observe their decision to cooperate.

Thus more generally, the screening function of rules may help explain a wide variety of observed practices in which groups impose costs on their members. Military organizations, religious orders, gangs, and other groups often require prospective members to make large sacrifices as part of the process of joining the group. Such costs are justified if they allow group leaders to identify cooperative types and screen out likely defectors. Moreover, by simply observing the willingness of others to follow rules, we have a mechanism by which we can easily identify prospective partners for any cooperative enterprise. 


\section{References}

AlgAZE, G. (2005): “The Sumerian Takeoff," Structure and Dynamics, 1(1).

Bellman, R. E. (1957): Dynamic Programming. Princeton University Press, Princeton, NJ. Republished in 2003: Dover.

Bru, L., S. Cabrera, C. M. Capra, and R. Gomez (2003): “A Common Pool Resource Game with Sequential Decisions and Experimental Evidence," Experimental Economics, 6, 91-114.

CÁrdenas, J.-C., M. JAnssen, And F. Bousquet (2011): “Dynamics of Rules and Resources: Three New Field Experiments on Water, Forests and Fisheries," in Handbook on Experimental Economics and the Environment, ed. by J. List, and M. Price. Edward Elgar Publishing.

CÁrdenAS, J.-C., And E. Ostrom (2004): "What do people bring into the game? Experiments in the field about cooperation in the commons," Agricultural Systems, $82,307-326$.

FALK, A., E. FEHR, AND U. FISCHBACHER (2002): "Appropriating the commons-a theoretical explanation," in The Drama of the Commons, ed. by R. Dietz, N. Dolšak, E. Ostrom, P. Stern, S. Stonich, and E. Weber, pp. 157-192. National Academy Press, Washington, DC.

FISCHBACHER, U. (2007): “z-Tree: Zurich Toolbox for Ready-made Economic Experiments," Experimental Economics, 10(2), 171-178.

FISCHBACHER, U., AND S. GÄCHTER (2010): "Social Preferences, Beliefs, and the Dynamics of Free Riding in Public Goods Experiments," American Economic Review, 100(1), 541-556.

FISCHBACHER, U., S. GÄCHTER, AND E. FEHR (2001): “Are people conditionally cooperative? Evidence from a public goods experiment," Economics Letters, 71(3), 397-404.

FudenberG, D., And J. Tirole (1991): Game Theory. MIT Press, Cambridge, MA.

GÄCHTER, S., B. HERRMANN, AND THÖNI (2010): “Culture and cooperation,” Philosophical transactions of the Royal Society of London. B, 365, 2651-2661.

GARDNer, R., E. Ostrom, And J. M. WAlKer (1990): “The Nature of Common-Pool Resource Problems," Rationality and Society, 2, 335-358.

GORDON, H. (1954): "The economic theory of a common-property resource: the fishery," The Journal of Political Economy, 62(2), 124-142.

Gunnthorsdottir, A., D. Houser, And K. MCCABE (2007): “Disposition, history and contributions in public goods experiments," Journal of Economic Behavior E Organization, 62(2), 304-315. 
HAIDT, J. (2007): “The New Synthesis in Moral Psychology," Science, 316(5827), 9981002.

HARDIN, G. (1968): "The tragedy of the commons," New York.

Henrich, J., R. Boyd, S. Bowles, C. CAMerer, E. Fehr, H. Gintis, R. McElreath, M. Alvard, A. Barr, J. Ensminger, N. Smith Henrich, K. Hill, F. Gil-White, M. Gurven, F. W. Marlowe, J. Q. Patton, and D. Tracer (2005): "Economic man" in cross-cultural perspective: Behavioral experiments in 15 small-scale societies," Behavioral and Brain Sciences, 28, 795-855.

Henrich, J., J. EnSMinger, R. MCElreath, A. BARR, C. BARRetT, A. Bolyanatz, J. C. Cardenas, M. Gurven, E. Gwako, N. Henrich, C. Lesorogol, F. Marlowe, D. Tracer, and J. Ziker (2010): “Markets, Religion, Community Size, and the Evolution of Fairness and Punishment," Science, 327(5972), 1480-1484.

Herrmann, B., C. ThÖNI, AND S. GÄChter (2008): “Antisocial Punishment Across Societies," Science, 319, 1362-1367.

Houser, D., M. KeAne, And K. MCCABe (2004): “Behavior in a dynamic decision problem: An analysis of experimental evidence using a Bayesian type classification algorithm," Econometrica, 72(3), 781-822.

IANNACCONE, L. (1992): "Sacrifice and stigma: reducing free-riding in cults, communes, and other collectives," Journal of Political economy, pp. 271-291.

Janssen, M. A. (2010): “Introducing Ecological Dynamics into Common-Pool Resource Experiments," Ecology and Society, 15(2), 7.

Janssen, M. A., J. M. Anderies, AND S. R. Joshi (2011): “Coordination and cooperation in asymmetric commons dilemmas," Experimental Economics, 14(4), 547-566.

Janssen, M. A., R. Holahan, A. Lee, And E. Ostrom (2010): “Lab Experiments for the Study of Social-Ecological Systems," Science, 328(5978), 613-617.

Kimbrough, E. O., And A. Vostroknutov (2012): “Rules, Rule-Following and Cooperation," Working Paper, Simon Fraser University.

KurzBAn, R., And D. Houser (2005): "Experiments Investigating Cooperative Types in Humans: A Complement to Evolutionary Theory and Simulations," Proceedings of the National Academy of Sciences of the United States of America, 102(5), 1803-1807.

LAMBA, S., AND R. MACE (2011): “Demography and ecology drive variation in cooperation across human populations," Proceedings of the National Academy of Sciences of the United States of America, 108(35), 14426-14430. 
McCabe, K., D. Houser, L. Ryan, V. Smith, and T. Trouard (2001): "A functional imaging study of cooperation in two-person reciprocal exchange," Proceedings of the National Academy of Sciences, 98(20), 11832.

Ostrom, E. (1990): Governing the Commons: the Evolution of Institutions for Collective Action, Political economy of institutions and decisions. Cambridge University Press, Cambridge.

_ (2009): "A general framework for analyzing sustainability of social-ecological systems," Science, 325(5939), 419-422.

(2010): "Beyond markets and states: polycentric governance of complex economic systems," American Economic Review, 100(3), 641-72.

Ostrom, E., R. Gardner, And J. Walker (1994): “Cooperation and Social Capital," in Rules, games, and common-pool resources, ed. by E. Ostrom, R. Gardner, and J. Walker. University of Michigan Press, Ann Arbor, MI.

Ostrom, E., J. Walker, And R. Gardner (1994): Rules, Games, and Common-Pool Resources. The University of Michigan Press, Ann Arbor, MI.

Rigdon, M. L., K. A. MCCABE, AND V. L. SMith (2007): “Sustaining Cooperation in Trust Games," The Economic Journal, 117(522), 991-1007.

Rodriguez-Sickert, C., R. A. GuZMÁN, AND J. C. CÁRdENAS (2008): “Institutions influence preferences: Evidence from a common pool resource experiment," Journal of Economic Behavior and Organization, 67, 215-227.

SMith, V. L. (2003): "Constructivist and ecological rationality in economics," The American Economic Review, 93(3), 465-508.

(2008): Rationality in Economics: Constructivist and Ecological Forms. New York, Cambridge University Press.

TANG, S. Y. (1994): "Institutions and Performance in Irrigation Systems," in Rules, Games and Common-Pool Resources, ed. by E. Ostrom, R. Gardner, and J. Walker, pp. 225-245. The University of Michigan Press, Ann Arbor, MI.

Velez, M. A., J. K. Stranlund, And J. J. Murphy (2009): “What motivates common pool resource users? Experimental evidence from the field," Journal of Economic Behavior and Organization, 70, 485-497.

WAlKer, J. M., AND R. GARDNeR (1992): "Probabilistic Destruction of Commonpool Resources: Experimental Evidence," The Economic Journal, 102(414), 11491161.

WALKER, J. M., R. GARDNER, AND E. Ostrom (1990): “Rent dissipation in a limitedaccess common-pool resource: Experimental evidence," Journal of Environmental Economics and Management, 19(3), $203-211$. 
Wilson, B. J., T. JAWORSKI, K. SCHURTER, AND A. SMYTH (2012): “The Ecological and Civil Mainsprings of Property: An Experimental Economic History of Whalers' Rules of Capture," Journal of Law, Economics and Organization, 28(4). 


\section{Appendix}

\section{A Proofs}

Proof of Proposition 1. If $w>n \bar{e}$ it is obvious that only the first case in the definition of $\pi_{i}$ can occur, or $\pi_{i}\left(e_{i}, e_{-i}\right)=\bar{e}+(\alpha-1) e_{i}$. Therefore, since $\alpha>1$, the only strictly undominated action is $e_{i}=\bar{e}$ for all $i$ which results in the proposed NE.

If $w \leq n \bar{e}$ then the following cases are possible. First, suppose that $E<w$, then $\pi_{i}\left(e_{i}, e_{-i}\right)=$ $\bar{e}+(\alpha-1) e_{i}$ for all players which implies that any player $i$ can profitably deviate by increasing her effort a little bit so that the sum of efforts is still less than $w$. Second, suppose that $E>w$, then $\pi_{i}\left(e_{i}, e_{-i}\right)=\bar{e}-e_{i}+\alpha \frac{w}{n}$ for all players which implies that any player $i$ can profitably deviate by decreasing her effort a little bit so that the sum of efforts is still higher than $w$. Therefore, in any NE it should be the case that $E=w$.

Now suppose that $E=w$. Then no player $i$ wishes to increase her effort as it would lower her payoff. However, it is possible that player $i$ might profitably deviate by decreasing her effort $e_{i}$ by small amount $\varepsilon$. In this case instead of payoff $\bar{e}-e_{i}+\alpha \frac{w}{n}$ she will get $\bar{e}+(\alpha-$ 1) $\left(e_{i}-\varepsilon\right)$. For this deviation to be unprofitable it must be the case that

$$
\bar{e}+(\alpha-1)\left(e_{i}-\varepsilon\right) \leq \bar{e}-e_{i}+\alpha \frac{w}{n} \quad \forall \varepsilon>0 .
$$

This inequality obviously holds if and only if $\bar{e}+(\alpha-1) e_{i} \leq \bar{e}-e_{i}+\alpha \frac{w}{n}$ or $e_{i} \leq \frac{w}{n}$.

Thus any NE should satisfy two conditions: 1) $E=w$; and 2) $e_{i} \leq \frac{w}{n}$ for all $i=1 . . n$. This is only possible if $e_{i}=\frac{w}{n}$ for all $i$.

$* * *$

Proof of Proposition 2. ${ }^{24}$ The proof consists of several cases. However let us make some observations beforehand. First, suppose that the amount of resource before some period is $w>n \bar{e}+\tau$. Then, even if all players choose maximal effort $\bar{e}$, the available amount of the resource at the beginning of the next period will actually be higher than in the previous period as long as $w-n \bar{e}+\beta(\bar{w}-(w-n \bar{e}))=(1-\beta)(w-n \bar{e})+\beta \bar{w} \geq w$. This can be rearranged to $w \leq \bar{w}-\frac{1-\beta}{\beta} n \bar{e}$. This means that for any values of the resource less than the right hand side even if all players choose maximal efforts the next period resource will be higher than original one. Notice that RHS can be negative (if $\beta$ or $\bar{w}$ is low). This means that for some combinations of parameters, the above statement will never hold. Second, for values $w>\bar{w}-\frac{1-\beta}{\beta} n \bar{e}$ if all players exert maximum effort, then the amount of the resource before next period will drop. However, if

$$
\bar{w}-\frac{1-\beta}{\beta} n \bar{e} \geq n \bar{e}+\tau
$$

then even if $w$ is above the threshold $\bar{w}-\frac{1-\beta}{\beta} n \bar{e}$, all players can exert full effort $\bar{e}$ and still have access to a resource level greater than $n \bar{e}+\tau$ next period. To see this rearrange inequality (4)

\footnotetext{
${ }^{24}$ In the proof we will slightly abuse notation. Remember that utility in each period of repeated game is defined in equation (1). Since in almost all cases in the proof we will only use the first part of the utility which is $\pi_{i}\left(e_{i}, \cdot\right)=\bar{e}+(\alpha-1) e_{i}$, we will drop $\bar{e}$ and $\alpha-1$ and think of utility as just being $\pi_{i}\left(e_{i}, \cdot\right)=e_{i}$. This does not change any results.
} 
to get

$$
\tau+\beta(\bar{w}-\tau) \geq n \bar{e}+\tau .
$$

The LHS of this inequality is the amount of resource available following periods in which the resource was harvested so that the remaining quantity was $\tau$, the minimum level at which the resource will regrow. This inequality says that even if the resource level reaches $\tau$, regrowth will ensure that enough of the resource is available in the following period for all players to exert the maximum effort, $\bar{e}$.

Case 1.1: $\bar{w}-\frac{1-\beta}{\beta} n \bar{e} \geq n \bar{e}+\tau$ and $w_{1} \geq n \bar{e}+\tau$.

As was described above, if before period $1 w_{1} \geq n \bar{e}+\tau$ then players can exert maximal efforts after all histories in all periods until the end of the game. Moreover, inequality (5) guarantees that after no history will the amount of resource available be below $n \bar{e}+\tau$. The strategy proposed in this Proposition has all players choosing $\bar{e}$ after each history. This is a SPNE since choosing $\bar{e}$ gives all players the maximum attainable payoff in each period.

Case 1.2: $\bar{w}-\frac{1-\beta}{\beta} n \bar{e} \geq n \bar{e}+\tau$ and $w_{1} \in[\tau, n \bar{e}+\tau)$.

If $w_{1} \in[\tau, n \bar{e}+\tau)$ then in period 1 players choose $e_{i 1}=\frac{w_{1}-\tau}{n}$ and then choose $\bar{e}$ in all consecutive periods. According to the "one-stage-deviation principle" (Fudenberg and Tirole, 1991, Theorem 4.1) we should only check for deviations in period 1 (again because in all other periods players have maximal payoffs). In period 1 player $i$ can increase that period's payoff by choosing effort $e_{i 1}=\frac{w_{1}-\tau}{n}+\varepsilon$, where $0<\varepsilon<\tau$. ${ }^{25}$ After this (and assuming all other players stick to the original strategy) the resource will drop to level $\tau-\varepsilon$, thus creating no growth afterwards. Therefore, following the strategy in all consecutive periods $i$ will get $\frac{w_{1}-\tau}{n}+\varepsilon+\frac{\tau-\varepsilon}{n}$. The last term here represents the payoff in second period. This deviation is not profitable as long as

$$
\frac{w_{1}-\tau}{n}+\varepsilon+\frac{\tau-\varepsilon}{n} \leq \frac{w_{1}-\tau}{n}+(L-1) \bar{e}
$$

To check whether this holds for all deviations $\varepsilon$ let us take a limit of the LHS to get the maximum possible deviation:

$$
\lim _{\varepsilon \uparrow \tau} \frac{w_{1}-\tau}{n}+\varepsilon+\frac{\tau-\varepsilon}{n}=\frac{w_{1}-\tau}{n}+\tau \leq \frac{w_{1}-\tau}{n}+(L-1) \bar{e}
$$

This holds whenever $\tau \leq(L-1) \bar{e}$ which is true by assumption of the Proposition. Therefore, the above strategy constitutes a SPNE.

Case 1.3: $\bar{w}-\frac{1-\beta}{\beta} n \bar{e} \geq n \bar{e}+\tau$ and $w_{1}<\tau$.

Finally if $w_{1}<\tau$ then no growth will happen in the game. In period 1 player $i$ exerts effort $\frac{w_{1}}{n}$. By choosing more effort she will be worse off in period 1 and still get 0 in all consecutive periods. She can also deviate by choosing less effort: $\frac{w_{1}}{n}-\varepsilon$. However, in this case her payoff will be $\frac{w_{1}}{n}-\varepsilon+\frac{\varepsilon}{n}$. Where the last term corresponds to the payoff in period 2. It is clear that this deviation is not profitable. Profitable deviations in periods other than 1 are impossible because $w_{2}=\ldots=w_{L}=0$. Thus we have a SPNE.

Next we analyze the case in which $\bar{w}-\frac{1-\beta}{\beta} n \bar{e}<n \bar{e}+\tau$. Notice that, analogously to inequality (5), we have now $\tau+\beta(\bar{w}-\tau)<n \bar{e}+\tau$. This implies that if after some period the

\footnotetext{
${ }^{25}$ Notice that decreasing effort in period 1 cannot increase player $i$ 's payoff since she will still get maximal utility from putting effort $\bar{e}$ in all following periods.
} 
amount of resource is $\tau$, then before next period the growth of the resource will be insufficient to allow all players to choose $\bar{e}$ without depleting the resource.

Case 2.1: $\bar{w}-\frac{1-\beta}{\beta} n \bar{e}<n \bar{e}+\tau$ and $w_{1} \in[\tau, n \bar{e}+\tau)$.

Assume that $w_{1} \in[\tau, n \bar{e}+\tau)$. Then, given the above observation, if players follow the strategy given in this Proposition they should choose effort $e_{i t}=\frac{w_{t}-\tau}{n}$ in periods 1 through $L-1$ and $e_{i L}=\min \left\{\bar{e}, \frac{w_{L}}{n}\right\}$ in the last period. Let us check that there are no possible one period deviations from this strategy. On the proposed path player $i$ gets payoff

$$
\frac{w_{1}-\tau}{n}+k \frac{(1-\beta) \tau+\beta \bar{w}-\tau}{n}+\min \left\{\bar{e}, \frac{(1-\beta) \tau+\beta \bar{w}}{n}\right\}
$$

where $k=L-2$.

Player $i$ might deviate by exerting less effort: $\frac{w_{1}-\tau}{n}-\varepsilon$. In this case new payoff is

$\frac{w_{1}-\tau}{n}-\varepsilon+\frac{(1-\beta)(\tau+\varepsilon)+\beta \bar{w}-\tau}{n}+(k-1) \frac{(1-\beta) \tau+\beta \bar{w}-\tau}{n}+\min \left\{\bar{e}, \frac{(1-\beta) \tau+\beta \bar{w}}{n}\right\}$.

This is a decreasing function of $\varepsilon$ which equals to the on-the-path payoff if $\varepsilon=0 .{ }^{26}$ Thus decreasing effort in period 1 is not profitable. This reasoning does not depend on number of periods $k$, therefore, the same logic can be applied to any period from 2 to $L-1$ to show that decreasing effort is not profitable. In period $L$ all players play NE of the stage game therefore no deviations can occur there either.

Let us now check if player $i$ could gain by increasing effort in period 1 . Deviation gives the payoff $\frac{w_{1}-\tau}{n}+\varepsilon+\frac{\tau-\varepsilon}{n}$ which is an increasing function of $\varepsilon$. Therefore the best deviation is given by the limit of this expression when $\varepsilon \rightarrow \tau$ which is

$$
\frac{w_{1}-\tau}{n}+\tau
$$

In order for the deviation to be unprofitable this must be less then or equal to the on-the-path payoff in expression (6) for all values of $k$. Expression (6) increases in $k$, therefore the most constraining case is $k=0$.

Deviation, thus, is unprofitable whenever (after rearranging given $k=0$ )

$$
\tau \leq \frac{\beta \bar{w}}{\beta+n-1} \quad \text { and } \quad \tau \leq \bar{e}
$$

Both inequalities hold by the assumption of the Proposition. Therefore we have a SPNE.

Case 2.2: $\bar{w}-\frac{1-\beta}{\beta} n \bar{e}<n \bar{e}+\tau$ and $w_{1} \geq n \bar{e}+\tau$ and small $L$.

Now let us assume that $w_{1} \geq n \bar{e}+\tau$. It is clear that at least in period 1 all players can exert effort $\bar{e}$ and not deplete the resource below level $\tau$. In general, by Lemma 1 with $W=n \bar{e}+\tau$ if all players exert effort $\bar{e}$ in all periods then there exists $M \in \mathbb{N}$ such that for all $k<M$, $w_{k} \geq n \bar{e}+\tau$ and for all $k \geq M, w_{k}<n \bar{e}+\tau$. Therefore, if $L<M$ then all players will receive the maximum payoff $\bar{e}$ in each period, which implies the absence of profitable deviations.

\footnotetext{
${ }^{26}$ If $\varepsilon$ is so big that before second period the resource is above $n \bar{e}-\tau$, then everyone will exert effort $\bar{e}$ which still makes this payoff function decreasing in $\varepsilon$.
} 
Thus, if $L<M$ then in SPNE all players put in effort $\bar{e}$ in all periods. ${ }^{27}$

Case 2.3: $\bar{w}-\frac{1-\beta}{\beta} n \bar{e}<n \bar{e}+\tau$ and $w_{1} \geq n \bar{e}+\tau$ and large $L$.

If, however, $L \geq M$ then there are periods at the end of the game in which the size of the resource falls below $n \bar{e}+\tau$ if all players exert effort $\bar{e}$ in periods before $M$ th. The strategy in this Proposition says that players should put in effort $\bar{e}$ in periods 1 through $M-1$; put in effort $\frac{w_{k}-\tau}{n}$ in periods $k=M . . L-1$; and effort $\min \left\{\bar{e}, \frac{w_{L}}{n}\right\}$ in period $L$. By Case 2.1 above we know that no single period deviations are possible in periods $M$ through $L$. Thus, it is only left to check single deviations in periods 1 to $M-1$.

First, let us make an observation. By Lemma 1 we know that in periods 1 to $M$ the size of the resource before period $t$ is defined by $w_{t}=(1-\beta) w_{t-1}+\beta c$, where $c=\bar{w}-\frac{1-\beta}{\beta} n \bar{e} .^{28}$ Suppose that in some period $t$ the amount of the resource increases by some $\varepsilon: w_{t}^{\prime}=w_{t}+\varepsilon$. Then in period $t+1$ the changed amount of the resource will be $w_{t+1}^{\prime}=(1-\beta) w_{t}^{\prime}+\beta c=$ $w_{t+1}+(1-\beta) \varepsilon$. Analogously, $w_{t+k}^{\prime}=w_{t+k}+(1-\beta)^{k} \varepsilon$. Therefore, an $\varepsilon$ increase in the resource in period $t$ increases the resource in period $t+k$ by $(1-\beta)^{k} \varepsilon$ as long as all players exert effort $\bar{e}$ in the interim periods.

Now consider a small reduction in effort in period $M-1$ by player $i$ keeping the prescribed strategy of the rest of the players fixed. Remember, by construction, $w_{M-1} \geq n \bar{e}+\tau$ and $w_{M}<n \bar{e}+\tau$. Suppose player $i$ decreases his effort in period $M-1$ from $\bar{e}$ to $\bar{e}-\varepsilon$ with very small $\varepsilon$. This, as described in the previous paragraph, will lead to change in $w_{M}$ : it will become $w_{M}+(1-\beta) \varepsilon$. Now, as long as $w_{M}+(1-\beta) \varepsilon \leq n \bar{e}+\tau$ the strategy prescribes all players to choose effort $\frac{w_{M}+(1-\beta) \varepsilon-\tau}{n}$. Since after this the resource level will fall to $\tau$, the introduction of small $\varepsilon$ will not have any effect on the payoffs in periods $M+1$ onwards. Therefore, $\varepsilon$ changes the overall payoff of player $i$ by $-\varepsilon+\frac{(1-\beta) \varepsilon}{n}<0$. So we might conclude that it is not worthwhile for player $i$ to decrease her effort by the amount $\varepsilon$ if $w_{M}+(1-\beta) \varepsilon \leq n \bar{e}+\tau$.

However, this is not enough to conclude that no single deviation in period $M-1$ is possible because a larger decrease in effort could also be profitable. For example, if player $i$ chooses $\varepsilon>\varepsilon_{1}$ where $\varepsilon_{1}$ satisfies $w_{M}+(1-\beta) \varepsilon_{1}=n \bar{e}+\tau$. As $\varepsilon>\varepsilon_{1}$ we still have resource level $w_{M}+(1-\beta) \varepsilon$ in period $M$. Now though $w_{M}+(1-\beta) \varepsilon>n \bar{e}+\tau$, which means that player $i$ in period $M$ should choose $\bar{e}$ regardless of $\varepsilon$. This in its turn implies that now $w_{M+1}$ will start changing to $w_{M+1}+(1-\beta)^{2} \varepsilon$. Overall change in payoff at $\varepsilon_{1}$ as was noticed above was $-\varepsilon_{1}+\frac{(1-\beta) \varepsilon_{1}}{n}<0$. As $\varepsilon$ grows above $\varepsilon_{1}$ the payoff in period $M$ stays fixed at $\bar{e}$, thus not influencing the difference in overall payoffs. However, the change in period $M+1$ now takes effect and creates an overall change in payoff for $\varepsilon>\varepsilon_{1}$ to be $-\varepsilon+\frac{(1-\beta)^{2} \varepsilon}{n}<0$ as long as $\varepsilon<\varepsilon_{2}$ which satisfies $w_{M+1}+(1-\beta) \varepsilon_{2}=n \bar{e}+\tau$. Thus a larger deviation resulting from choosing $\varepsilon$ above $\varepsilon_{1}$ is even less profitable.

We can extend this argument and construct $\varepsilon_{3}, \varepsilon_{4}$, etc. tracking the change in overall payoff to see that the more $\varepsilon$ increases the more negative the overall change in payoff becomes. Therefore we can conclude that decreasing effort in period $M-1$ for player $i$ is not profitable.

Now suppose that player $i$ decreases effort in period $M-2$ and it becomes $\bar{e}-\varepsilon$. As a result, the resource level in period $M-1$ will become $w_{M-1}+(1-\beta) \varepsilon$. But since in period $M-1$ player $i$ already exerts effort $\bar{e}$, an increase in the resource level will have no effect on the payoff in period $M-1$. However, an $\varepsilon$ change in $M-2$ will increase the resource size to $w_{M}+(1-\beta)^{2} \varepsilon$ in period $M$. Using the argument developed above, for $\varepsilon \leq \varepsilon_{1}^{\prime}$ which

\footnotetext{
${ }^{27}$ See Lemma 1 for the exact formula for $M$ in terms of the parameters of the model.
}

${ }^{28}$ If players stick to the strategy prescribed by this Proposition. 
satisfies $w_{M}+(1-\beta)^{2} \varepsilon_{1}^{\prime}=n \bar{e}+\tau$, the overall change in payoff will be $-\varepsilon+\frac{(1-\beta)^{2} \varepsilon}{n}<0$. For $\varepsilon>\varepsilon_{1}^{\prime}$ the argument goes exactly as before and demonstrates that in period $M-2$ it is also not profitable to deviate from the prescribed strategy. Analogous arguments can be given for all periods $k=1 . . M-3$.

We showed that there exists no single period deviation in any period by which player $i$ can profitably deviate from the strategy given in the Proposition. Therefore we have a SPNE.

Case 2.4: $\bar{w}-\frac{1-\beta}{\beta} n \bar{e}<n \bar{e}+\tau$ and $w_{1}<\tau$.

Same as Case 1.3.

$$
* * *
$$

Proof of Proposition 3. Some observation first. Notice that the restrictions on $\tau$ in this Proposition imply

$$
(1-\beta) \tau+\beta \bar{w} \leq n \tau \leq n \bar{e}
$$

Moreover, the following equivalence holds:

$$
(1-\beta) \tau+\beta \bar{w} \leq n \bar{e} \Longleftrightarrow \bar{w}-\frac{1-\beta}{\beta} n \bar{e} \leq n \bar{e}-\frac{1-\beta}{\beta} \tau<n \bar{e} .
$$

Case $w_{1} \in[n \tau, n \bar{e})$.

Here $w_{1} \geq n \tau$ which implies that one player can deviate from the strategy described in the Proposition (choose $\frac{w_{1}}{n}$ ) so that resource is depleted to the level at least $\tau$. On (proposed) equilibrium path payoff in this case is $\frac{w_{1}}{n}$ and the deviation payoff is $\frac{w_{1}}{n}-(\tau+\varepsilon)+\frac{(1-\beta)(\tau+\varepsilon)+\beta \bar{w}}{n}$. Here one player exerts effort $\frac{w_{1}}{n}-(\tau+\varepsilon)$ instead of prescribed $\frac{w_{1}}{n}$. It is clear that deviation payoff decreases in $\varepsilon$, thus the best deviation payoff is $\frac{w_{1}}{n}-\tau+\frac{(1-\beta) \tau+\beta \bar{w}}{n}$. Notice that due to (8) players can deplete the resource to zero in the second period after deviation, so no min operator on $\frac{(1-\beta) \tau+\beta \bar{w}}{n}$ is required. No deviation occurs if (after rearranging) $\tau \geq \frac{\beta \bar{w}}{\beta+n-1}$, exactly as assumed in the Proposition. Since the resource is depleted after at most 2 periods the same logic can be used for deviations in any period $t=1 . . L-1$ as long as $w_{t} \in[n \tau, n \bar{e})$.

Case $w_{1} \in(0, n \tau)$.

Here no player can deviate in period 1 so that the resource reaches the level where it can grow next period. Exerting $\varepsilon$ less effort in period 1 gives payoff $\frac{w_{1}}{n}-\varepsilon+\frac{\varepsilon}{n}$. This is decreasing in $\varepsilon$, thus no profitable deviation is possible. The same logic works in any period $t=1 . . L-1$ with $w_{t} \in(0, n \tau)$.

Case $w_{1} \in[n \bar{e}, \bar{w}]$.

This case is analogous to Cases 2.2 and 2.3 of Proposition 2. Notice first that equivalence (9) guarantees that $\bar{w}-\frac{1-\beta}{\beta} n \bar{e}<n \bar{e}$. Thus, by Lemma 1 with $W=n \bar{e}$ if all players exert effort $\bar{e}$ in all periods then there exists $M \in \mathbb{N}$ such that for all $k<M, w_{k} \geq n \bar{e}$ and for all $k \geq M, w_{k}<n \bar{e}$. Therefore, if $L<M$ then all players will receive the maximum payoff $\bar{e}$ in each period, which implies the absence of profitable deviations. If $L \geq M$ then the same construction as in Case 2.3 of Proposition 2 (together with two cases above in this proof) guarantees the absence of profitable deviations.

Proof of Proposition 4. We employ an induction argument on the sequence of value func- 
tions constructed by working backwards from the last period. Let us restate the maximization problem:

$$
\begin{array}{ll} 
& \max _{\left(e_{t}\right)_{t=1 . L}} \sum_{t=1}^{L} e_{t} \\
\text { s.t. } & w_{t+1}=(1-\beta)\left(w_{t}-e_{t}\right)+\beta \bar{w} \\
& 0 \leq e_{t} \leq \min \left\{\bar{e}, w_{t}-\tau\right\} \text { for } t=1 . . L-1 \\
& 0 \leq e_{L} \leq \min \left\{\bar{e}, w_{L}\right\} .
\end{array}
$$

Consider the value function which by Bellman's Principle of Optimality (Bellman, 1957) should characterize the solution in period $L$ :

$$
\begin{aligned}
& V_{L}(w)=\max _{e} e \\
& \text { s.t. } 0 \leq e \leq \min \{\bar{e}, w\} .
\end{aligned}
$$

Obviously, $V_{L}(w)=\min \{\bar{e}, w\}$ which implies that $V_{L}(w)=\bar{e}$ if $w>\bar{e}$ and $V_{L}(w)=w$ if $w \leq \bar{e}$. Thus, $V_{L}$ is a weakly increasing piecewise linear continuous function with slope 0 or 1 .

Now consider the value function $V_{L-1}:[\tau, \bar{w}] \rightarrow \mathbb{R}$ for period $L-1$ :

$$
\begin{aligned}
V_{L-1}(w)= & \max _{e} e+V_{L}\left(w^{\prime}\right) \\
\text { s.t. } & w^{\prime}=(1-\beta)(w-e)+\beta \bar{w} \\
& 0 \leq e \leq \min \{\bar{e}, w-\tau\} .
\end{aligned}
$$

For any fixed $w$ the maximand $e+V_{L}((1-\beta)(w-e)+\beta \bar{w})$ is an increasing piecewise linear continuous function of $e$ because $V_{L}((1-\beta)(w-e)+\beta \bar{w})$ is a decreasing function of $e$ with slopes $-(1-\beta)$ or 0 . Thus, the solution to the maximization problem is given by the maximum $e$ possible. Therefore, if $w \leq \bar{e}+\tau$, we have $V_{L-1}(w)=w-\tau+\min \left\{\bar{e}_{,}(1-\beta) \tau+\beta \bar{w}\right\}$, and if $w>\bar{e}+\tau$, then $V_{L-1}(w)=\bar{e}+\min \{\bar{e},(1-\beta)(w-\bar{e})+\beta \bar{w}\}$. Plugging $w=\bar{e}+\tau$ into these two definitions we can easily see that $V_{L-1}$ connects at $V_{L-1}(\bar{e}+\tau)=\bar{e}+\min \{\bar{e},(1-$ $\beta) \tau+\beta \bar{w}\}$. Since both definitions of $V_{L-1}$ are weakly increasing we can conclude that $V_{L-1}$ is weakly increasing continuous piecewise linear with slopes 1, 0 and possibly $1-\beta$ (if $\bar{e}>$ $(1-\beta)(w-\bar{e})+\beta \bar{w})$. Notice that the optimal $e$ here is the same as described in this Proposition.

Now we are ready to formulate the induction argument. $V_{L-2}$ is given by

$$
\begin{aligned}
V_{L-2}(w)= & \max _{e} e+V_{L-1}\left(w^{\prime}\right) \\
\text { s.t. } \quad & w^{\prime}=(1-\beta)(w-e)+\beta \bar{w} \\
& 0 \leq e \leq \min \{\bar{e}, w-\tau\} .
\end{aligned}
$$

Again, $e+V_{L-1}((1-\beta)(w-e)+\beta \bar{w})$ is an increasing function of $e$, thus the optimal $e$ is the maximum one. ${ }^{29}$ If $w \leq \bar{e}+\tau$, we have $V_{L-2}(w)=w-\tau+V_{L-1}((1-\beta) \tau+\beta \bar{w})$, and if $w>\bar{e}+\tau$, then $V_{L-2}(w)=\bar{e}+V_{L-1}((1-\beta)(w-\bar{e})+\beta \bar{w})$. Again, the two pieces of $V_{L-2}$ connect at $V_{L-2}(\bar{e}+\tau)=\bar{e}+V_{L-1}((1-\beta) \tau+\beta \bar{w})$, and since both pieces are weakly increasing and continuous, we conclude that $V_{L-2}$ is weakly increasing continuous piecewise

\footnotetext{
${ }^{29}$ This is because the slopes of $V_{L-1}$ do not exceed 1.
} 
linear with slopes: 1 (first piece) and some slopes that do not exceed $1-\beta$ (second piece).

Notice that in this argument we used only two properties of $V_{L-1}: 1$ ) that it is weakly increasing continuous; 2 ) that it is piecewise linear with slopes not exceeding 1 . Thus, since we found that $V_{L-2}$ enjoys both of these properties, the same argument can be used to show that for any $k>2$

$$
\begin{aligned}
V_{L-k}(w)= & \max _{e} e+V_{L-k+1}\left(w^{\prime}\right) \\
\text { s.t. } & w^{\prime}=(1-\beta)(w-e)+\beta \bar{w} \\
& 0 \leq e \leq \min \{\bar{e}, w-\tau\}
\end{aligned}
$$

is a weakly increasing continuous piecewise linear with slopes not exceeding 1 . By the Principle of Optimality the sequence of functions $\left(V_{t}\right)_{t=1 . . L}$ characterizes the solution to the problem (3) and consecutively to the problem (2) if $\tau<\bar{e}$. This, in its turn, implies that choices of $e_{t}$ are as described in the Proposition.

\section{B Lemmata}

Lemma 1. Suppose $c:=\bar{w}-\frac{1-\beta}{\beta} n \bar{e}<W$ and $w_{1} \geq W$. If in each period $k \geq 1$ all players exert effort $\bar{e}$ then there exists $M \in \mathbb{N}$ such that for all $k<M, w_{k} \geq W$ and for all $k \geq M, w_{k}<W$. Moreover, $M=1+\left\lceil\log _{1-\beta} \frac{W-c}{w_{1}-c}\right\rceil$.

Proof. Suppose before period 1 the amount of the resource is $w_{1}$. If all players exert effort $\bar{e}$ then before period 2 the amount of resource will be

$$
w_{2}=(1-\beta)\left(w_{1}-n \bar{e}\right)+\beta \bar{w}=(1-\beta) w_{1}+\beta\left[\bar{w}-\frac{1-\beta}{\beta} n \bar{e}\right] .
$$

Analogously, if players continue exerting effort $\bar{e}$, the amount of resource at the beginning of period $t$ will be

$$
w_{t}=(1-\beta) w_{t-1}+\beta\left[\bar{w}-\frac{1-\beta}{\beta} n \bar{e}\right] .
$$

Let $c=\bar{w}-\frac{1-\beta}{\beta} n \bar{e}$. Then we can rewrite the difference equation above as $w_{t}=(1-\beta) w_{t-1}+$ $\beta c$. This difference equation has a unique solution of the form

$$
w_{t}=C(1-\beta)^{t}+c
$$

where $C=\frac{w_{1}-c}{1-\beta}$ depends on the initial conditions (in our case $w_{1}$ ). By assumption of the Lemma $C>0$. It is clear that $\lim _{t \rightarrow \infty} w_{t}=c<W$. Therefore, there exists a period $M$ such that $w_{M}<W$. Moreover, $w_{t}$ is strictly decreasing sequence, thus the conditions of the Lemma are satisfied. To find $M$ solve for $t$ in $C(1-\beta)^{t}+c=W$. It gives

$$
M=1+\left\lceil\log _{1-\beta} \frac{W-c}{w_{1}-c}\right\rceil .
$$


The logarithm is well defined by the assumptions of the Lemma.

$$
* * *
$$

Lemma 2. For any solution to Social Planner's problem (2):

$$
\begin{array}{ll} 
& \max _{\left(e_{t}\right)_{t=1 . . L}} \sum_{t=1}^{L} e_{t} \\
\text { s.t. } & w_{t+1}=w_{t}-e_{t}+\beta\left(\bar{w}-\left(w_{t}-e_{t}\right)\right) \mathbb{1}_{w_{t}-e_{t} \geq \tau} \\
& 0 \leq e_{t} \leq \min \left\{\bar{e}, w_{t}\right\}
\end{array}
$$

it is true that $w_{t} \geq \tau$ for all $t=1 . . L-1$ whenever $w_{1} \geq \tau$ and $\tau<\bar{e}$.

Proof. Suppose $\left(e_{t}\right)_{t=1 . . L}$ is a solution to the problem (2) and suppose that for some $k \leq L-1$ the size of the resource is $w_{k} \geq \tau$ and $w_{k+1}<\tau$. By condition (10) this implies that $w_{t}<\tau$ for all future periods $t>k+1$ as resource growth is not possible anymore. For any solution $\left(e_{t}\right)_{t=1 . . L}$, it will then be true that

$$
\sum_{t=k+1}^{L} e_{t}=w_{k+1}
$$

as "eating" less than that amount would be not optimal. This also implies that $\sum_{t=k}^{L} e_{t}=w_{k}$ (by (10)).

Suppose now that a player exerts effort $e_{k}=w_{k}-\tau$ instead of $w_{k}-w_{k+1}$ in period $k$ and in period $k+1$ she consumes as much as possible: $\min \{\bar{e},(1-\beta) \tau+\beta \bar{w}\}$. Then the payoff from period $k$ on is at least $w_{k}-\tau+\min \{\bar{e},(1-\beta) \tau+\beta \bar{w}\}$, which is $w_{k}+\bar{e}-\tau>w_{k}$ or $w_{k}+\beta(\bar{w}-\tau)>w_{k}$. In both cases this is higher than the original continuation payoff $w_{k}$. Therefore, it cannot be optimal to exert effort $w_{k}-w_{k+1}$ in period $k$ reducing size of the resource below $\tau$.

\section{Social Planner's Problem with a Negative Externality}

In this section we consider a modified Social Planner's problem. We assume that when the resource is depleted below some level, continuing to harvest the resource imposes a negative externality. We assume that there is a level $\eta>\tau$ such that if in period $t$ the resource is above $\eta$ then society's utility in period $t$ becomes $e_{t}+u$ where $u>0$ represents the benefit to the society from having sufficient resource stock to satisfy non-harvesting individuals. Thus Social Planner's problem is then:

$$
\begin{array}{ll} 
& \max _{\left(e_{t}\right)_{t=1 . . L}} \sum_{t=1}^{L} e_{t}+u \mathbb{1}_{w_{t} \geq \eta} \\
\text { s.t. } & w_{t+1}=w_{t}-e_{t}+\beta\left(\bar{w}-\left(w_{t}-e_{t}\right)\right) \mathbb{1}_{w_{t}-e_{t} \geq \tau} \\
& 0 \leq e_{t} \leq \min \left\{\bar{e}, w_{t}\right\} .
\end{array}
$$


Since by assumption $\eta>\tau$, Lemma 2 applies to this maximization problem exactly as it did to the problem (2). Therefore, we can rewrite problem (11) as

$$
\begin{array}{ll} 
& \max _{\left(e_{t}\right)_{t=1 . L}} \sum_{t=1}^{L} e_{t}+u \mathbb{1}_{w_{t} \geq \eta} \\
\text { s.t. } & w_{t+1}=(1-\beta)\left(w_{t}-e_{t}\right)+\beta \bar{w} \\
& 0 \leq e_{t} \leq \min \left\{\bar{e}_{,} w_{t}-\tau\right\} \text { for } t=1 . . L-1 \\
& 0 \leq e_{L} \leq \min \left\{\bar{e}, w_{L}\right\} .
\end{array}
$$

Let $w^{*}=\frac{\eta-\beta \bar{w}}{1-\beta}+\bar{e}$ and $w_{*}=\frac{\eta-\beta \bar{w}}{1-\beta}$. Then the following Proposition characterizes the solution to problem (11).

Proposition 5. Suppose that $\tau<w^{*}<\bar{w}$ and $\tau<w_{*}{ }^{30}$ The choice procedure that generates the optimal solution in problem (11) is the same as in problem (2) except that in each period $t=1 . . L-1$ there exists a non-empty interval $\left[a_{t}, w^{*}\right]$ with $a_{t} \geq \tau$ such that $e_{t}=w_{t}-w_{*}$ for all $w_{t} \in\left[a_{t}, w^{*}\right]$.

Proof. See below in this Appendix.

Notice that removing two assumptions of the Proposition $\left(\tau<w^{*}<\bar{w}\right.$ and $\left.\tau<w_{*}\right)$ does not change the final conclusions regarding the optimal choice procedure. The only difference is that without these assumptions some parts of the proof become unnecessary.

Now we will characterize what happens on the optimal path of problem (11). Proposition 5 says that on the optimal path, maximal effort $\bar{e}$ or the effort that reduces the next period resource stock to $(1-\beta) \tau+\beta \bar{w}$ will be chosen in all periods with the exception of those periods in which the previous period's resource stock lies in the interval $w_{t} \in\left[a_{t}, w^{*}\right]$. In those cases, effort is chosen so that the next period resource level is $\eta$. Since $\left(a_{t}\right)_{t=1 . . L-1}$ depend on the exact shapes of the value functions in each period, it is hard to provide an exact formula for each $a_{t}$. However, it is possible to estimate maximal values of $a_{t}$. Consider equation (15) from the proof:

$$
v_{t-1}(w)=\max \left\{w-\frac{\eta-\beta \bar{w}}{1-\beta}+V_{t}(\eta), \bar{e}+V_{t}((1-\beta)(w-\bar{e})+\beta \bar{w})\right\} .
$$

It characterizes the continuous part of the value function in the interval of resource levels $w \in\left[w_{*}, w^{*}\right)$. We know from the proof that $a_{t}$ is either the resource level where two functions inside the max operator intersect or is equal to $w_{*}$. Therefore, the minimum possible $a_{t}$ is $w_{*}=w^{*}-\bar{e}$ and the maximum possible $a_{t}$ is attained if $\bar{e}+V_{t}((1-\beta)(w-\bar{e})+\beta \bar{w})$ is constant in $w$ and intersects $w-\frac{\eta-\beta \bar{w}}{1-\beta}+V_{t}(\eta)$. Inequality (16) then shows that maximal $a_{t}$ is $w^{*}-u$. Therefore, in any period $t$ the length of the interval $\left[a_{t}, w^{*}\right]$ is $\min \{u, \bar{e}\} .{ }^{31}$

The length of the interval $\left[a_{t}, w^{*}\right]$ (in which the optimal choice is to harvest the resource so that the next period's resource stock is equal to $\eta$ ) is important for the optimal path that will be followed. If this interval is sufficiently small (e.g. if $u \rightarrow 0$ ), then the optimal path will be very similar to the path of problem (2). On the other hand, if the length is $\bar{e}$, then the optimal

\footnotetext{
${ }^{30}$ These assumptions guarantee that the most general case of the problem is considered.

${ }^{31}$ However, depending on the parameters it may be the case that $w^{*}$ is very close to $\tau$. In this case $a_{t}=\tau$ and the length of the interval is $w^{*}-\tau$. In interesting cases though we will have $a_{t}>\tau$ and the length will be $\min \{u, \bar{e}\}$.
} 
path will converge to $\eta$ from some period until the end of the game as long as initial $w_{1}$ is big enough. To see this consider three cases.

Case $(1-\beta) \tau+\beta \bar{w}>\eta$ and $u>0$

Here $\eta$ is so small that even if the resource falls to the level $\tau$, next period it will regrow to be larger than $\eta$. This implies that whatever the initial conditions are, the resource stock will always remain above $\eta$.

Case $(1-\beta) \tau+\beta \bar{w} \in\left[w_{*}, \eta\right]$ and $u \geq \bar{e}$

Here the optimal path will either reduce the resource level to $\eta$ and remain there until the last period or will at some point exceed $\eta$ and remain greater than $\eta$ until the last period. Notice first that $u \geq \bar{e}$ implies that in any period the length of the interval $\left[a_{t}, w^{*}\right]$ is $\bar{e}$ (or $a_{t}=w_{*}$ ). Also notice that even if the resource falls to $\tau$ after some period it will grow to $(1-\beta) \tau+\beta \bar{w}>w_{*}$ next period thus necessitating the jump to $\eta$ in the subsequent period.

If $\eta \in\left(w_{*}, w^{*}\right]$ then

$$
(1-\beta) \tau+\beta \bar{w} \leq \eta \Leftrightarrow \eta-e^{*}(\eta) \geq \tau
$$

where $e^{*}(\eta)=\eta-w_{*}$ is the optimal choice at $\eta$. This equivalence means that once the level of the resource is $\eta$, it will remain there since at this level, the optimal choice is feasible. The resource stock will always end up at level $\eta$ at some point and the planner will continue to harvest so that it remains there. This is because: 1$)$ for $w_{1} \in\left[\tau, w_{*}\right)$, the next period resource level will be $(1-\beta) \tau+\beta \bar{w} \in\left[w_{*}, \eta\right]$ and thus $\eta$ in the subsequent period; 2$)$ for $w_{1} \in\left[w_{*}, w^{*}\right]$, the next period resource level will be $\eta$; 3) for $w_{1}>w^{*}$ the resource level will eventually be reduced into the interval $\left(w_{*}, w^{*}\right]$ since

$$
\eta \leq w^{*} \Leftrightarrow \eta \geq \bar{w}-\frac{1-\beta}{\beta} \bar{e} \Leftrightarrow w^{*} \geq \bar{w}-\frac{1-\beta}{\beta} \bar{e},
$$

and $\bar{w}-\frac{1-\beta}{\beta} \bar{e}$ is the precise resource level such that, by exerting effort $\bar{e}$ above it, the planner will always reduce the next period resource level, so that it converges to $\bar{w}-\frac{1-\beta}{\beta} \bar{e}$ (see Lemma 1 with $n=1)$.

If $\eta>w^{*}$ then at some point, the resource level will increase above $\eta$ and will remain above it until the final period. Indeed,

$$
\eta>w^{*} \Leftrightarrow \eta<\bar{w}-\frac{1-\beta}{\beta} \bar{e} .
$$

This means that for $w_{1} \in[\tau, \eta]$ the resource stock will increase towards $\bar{w}-\frac{1-\beta}{\beta} \bar{e}$, necessarily passing through the interval $\left[w_{*}, w^{*}\right]$, since its length is $\bar{e}$. This implies that the resource stock will be equal to $\eta$ at some point. Once $\eta$ is reached, the optimal choice will be $e^{*}(\eta)=\bar{e}$. But by definition of $w^{*}$ (see proof of Proposition 5) for any resource $w>w^{*}$ even if effort $\bar{e}$ is exerted, the next period resource level will be greater than $\eta$. Thus, since $\eta>w^{*}$ at some point, the resource level will always remain greater than $\eta$. Thus, for $w_{1}>\eta>w^{*}$ the next period resource (and all others until the end) will remain above $\eta$ for the same reason.

Case $(1-\beta) \tau+\beta \bar{w}<w_{*}$ and $u \geq \bar{e}$

In this case the resource level will either drop to $(1-\beta) \tau+\beta \bar{w}$ or behave as in the previous case. As before we have $a_{t}=w_{*}$ for all $t$ and, since $(1-\beta) \tau+\beta \bar{w}<w_{*}$, if $w_{1} \in\left[\tau, w_{*}\right)$ the resource will stay at level $(1-\beta) \tau+\beta \bar{w}<w_{*}<\eta$ until the end of the game (last inequality is true since $w_{*}<\eta \Leftrightarrow \eta<\bar{w}$ always holds). 
If $\eta \in\left(w_{*}, w^{*}\right]$ then

$$
(1-\beta) \tau+\beta \bar{w}<w_{*} \Rightarrow \eta-e^{*}(\eta)>\tau
$$

where $e^{*}(\eta)=\eta-w_{*}$. Thus, as before, if resource falls into the interval $\left(w_{*}, w^{*}\right]$ it will stay at level $\eta$ until last period. The rest of the argument for $\eta \in\left(w_{*}, w^{*}\right]$ and $\eta>w^{*}$ is the same as in the previous case excluding values $w_{1} \in\left[\tau, w_{*}\right)$.

\section{C.1 Proof of Proposition 5}

Proof of Proposition 5. The proof follows the same logic as the proof of Proposition 4. Let us restate the maximization problem:

$$
\begin{array}{ll} 
& \max _{\left(e_{t}\right)_{t=1 . L}} \sum_{t=1}^{L} e_{t}+u \mathbb{1}_{w_{t} \geq \eta} \\
\text { s.t. } & w_{t+1}=(1-\beta)\left(w_{t}-e_{t}\right)+\beta \bar{w} \\
& 0 \leq e_{t} \leq \min \left\{\bar{e}, w_{t}-\tau\right\} \text { for } t=1 . . L-1 \\
& 0 \leq e_{L} \leq \min \left\{\bar{e}, w_{L}\right\} .
\end{array}
$$

The value function that characterizes the solution in period $L$ is

$$
\begin{aligned}
V_{L}(w)= & \max _{e} e+u \mathbb{1}_{w \geq \eta} \\
\text { s.t. } & 0 \leq e \leq \min \{\bar{e}, w\} .
\end{aligned}
$$

Clearly, $V_{L}(w)=\min \{\bar{e}, w\}$ for $w<\eta$ and $V_{L}(w)=\min \{\bar{e}, w\}+u$ for $w \geq \eta . V_{L}(w)$ is piecewise linear weakly increasing function with slopes 1 and 0 and with discontinuity at $w=\eta$. Let us write $V_{L}(w)=v_{L}(w)+u \mathbb{1}_{w \geq \eta}$ where $v_{L}(w)=\min \{\bar{e}, w\}$ is continuous "part" of $V_{L} \cdot{ }^{32}$ Notice that $v_{L}$ is equal to the analogous function in the proof of Proposition 4. Therefore, the choice procedure prescribed by $V_{L}$ is the same as in Proposition 4 ( $u \mathbb{1}_{w \geq \eta}$ doesn't play any role here).

Consider now the value function $V_{L-1}:[\tau, \bar{w}] \rightarrow \mathbb{R}$ for period $L-1$ :

$$
\begin{aligned}
V_{L-1}(w)= & \max _{e} e+u \mathbb{1}_{w \geq \eta}+V_{L}\left(w^{\prime}\right) \\
\text { s.t. } \quad & w^{\prime}=(1-\beta)(w-e)+\beta \bar{w} \\
& 0 \leq e \leq \min \{\bar{e}, w-\tau\} .
\end{aligned}
$$

Here $u \mathbb{1}_{w \geq \eta}$ is constant for each given $w$. Therefore, the function

$$
\begin{aligned}
v_{L-1}(w)= & \max _{e} e+V_{L}\left(w^{\prime}\right) \\
\text { s.t. } & w^{\prime}=(1-\beta)(w-e)+\beta \bar{w} \\
& 0 \leq e \leq \min \{\bar{e}, w-\tau\} .
\end{aligned}
$$

is a candidate for the continuous part of $V_{L-1}$.

Now we will show two things simultaneously: 1) that $v_{L-1}$ is continuous and 2) that optimal choices of effort for different $w$ correspond to those described in this Proposition. Con-

${ }^{32}$ The notation $V_{t}(w)=v_{t}(w)+u \mathbb{1}_{w \geq \eta}$ will be used throughout the proof. 
sider first the levels of the resource $w$ satisfying $(1-\beta)(w-\bar{e})+\beta \bar{w} \geq \eta$. We can rearrange this to get $w \geq \frac{\eta-\beta \bar{w}}{1-\beta}+\bar{e}=w^{*}{ }^{33}$ For all these levels $w \geq w^{*}$ it is true that if $w$ is the amount of the resource before period $L-1$, then, even if maximal effort $\bar{e}$ is exerted, in period $L$ the amount of the resource will be higher than $\eta$. This implies that $V_{L}(w)=v_{L}(w)+u$ for all $w \geq w^{*}$ in problem (13). This in turn means that for all $w \geq w^{*}$ the optimal choices of effort will coincide with analogous choices in the proof of Proposition 4: all that changes is added constant $u$. Moreover, $v_{L-1}(w)$ restricted to $w \geq w^{*}$ is continuous by Proposition 4 .

Using the same argument, consider levels of the resource $w$ such that $(1-\beta) w+\beta \bar{w}<\eta$. These levels $w$ are such that even if no effort is exerted in the current period, next period the amount of the resource will be less than $\eta$. We can rewrite this as $w<\frac{\eta-\beta \bar{w}}{1-\beta}=w_{*} \cdot{ }^{34}$ For all $w \in\left[\tau, w_{*}\right)$ it is then true that $V_{L}(w)=v_{L}(w)$. Thus, for all $w \in\left[\tau, w_{*}\right)$ the optimal choices are the same as in the analogous problem in Proposition 4 . Moreover, $v_{L-1}$ restricted to $w \in\left[\tau, w_{*}\right)$ is continuous by Proposition 4 .

Now let us find optimal choices for $w \in\left[w_{*}, w^{*}\right)$. First, consider the maximand function $e+V_{L}\left(w^{\prime}\right)$ in (13) when $w=w^{*}$. For $w=w^{*}, w^{\prime}$ ranges in the interval $\left[\eta,(1-\beta) w^{*}+\beta \bar{w}\right]$ as $e$ changes from 0 to $\bar{e}^{35}$ Thus, $e+V_{L}\left((1-\beta)\left(w^{*}-e\right)+\beta \bar{w}\right)$ is a continuous, strictly increasing function of $e$ as $V_{L}$ restricted to $\left[\eta,(1-\beta) w^{*}+\beta \bar{w}\right]$ is weakly increasing with slopes no more than 1 and continuous. Now let us perform the same analysis for $w=w^{*}-\varepsilon$ where $\varepsilon$ is sufficiently small positive number. For $w=w^{*}-\varepsilon, w^{\prime}$ ranges in the interval $[\eta-(1-\beta) \varepsilon,(1-$ $\left.\beta) w^{*}+\beta \bar{w}-(1-\beta) \varepsilon\right]$ as $e$ changes. Thus, the maximand function

$$
m(e ; \varepsilon)=e+V_{L}\left((1-\beta)\left(w^{*}-\varepsilon-e\right)+\beta \bar{w}\right)
$$

increases strictly and continuously as $e$ goes from 0 to $\bar{e}-\varepsilon$, then has a discontinuous drop of size $(1-\beta) u$, and then increases again strictly and continuously as $e$ goes from $\bar{e}-\varepsilon$ to $\bar{e}^{36}$ Given $\varepsilon$ small enough the optimal effort choice will be at $e^{*}=\bar{e}-\varepsilon$ or such that $(1-$ $\beta)\left(w^{*}-\varepsilon-e^{*}\right)+\beta \bar{w}=\eta$ which gives $e^{*}=w^{*}-\varepsilon-\frac{\eta-\beta \bar{w}}{1-\beta}=w-\frac{\eta-\beta \bar{w}}{1-\beta}$ as described in the Proposition. More importantly though, this argument shows the existence of an interval $\left[a_{L-1}, w^{*}\right)$ of levels of the resource (some range of small enough $\varepsilon$ ) such that $e^{*}=w-\frac{\eta-\beta \bar{w}}{1-\beta}$ for all $w \in\left[a_{L-1}, w^{*}\right)$. Notice that this implies that effort $e^{*}$ in this interval is chosen so that the amount of the resource before period $L$ is exactly $\eta$. It is also easy to observe that $v_{L-1}(w)$ is continuous on the interval $\left(w^{*}-\varepsilon, w^{*}+\varepsilon\right)$ as $\lim _{\varepsilon \rightarrow 0} e^{*}=\bar{e}$.

Before continuing let us make an observation about the function $m(e ; \varepsilon)$. It consists of two increasing continuous functions with a discontinuous drop at $e=\bar{e}-\varepsilon$. This implies that the maximum of $m(e ; \varepsilon)$ can occur in only two places: at $e=\bar{e}-\varepsilon$ or at $e=\bar{e}$. As shown above, this implies that for $w=w^{*}-\varepsilon$ we have either $e^{*}=w-\frac{\eta-\beta \bar{w}}{1-\beta}$ or $e^{*}=\bar{e}$ on the interval $w \in\left[w_{*}, w^{*}\right)$. Thus we can rewrite $v_{L-1}(w)$ on $w \in\left[w_{*}, w^{*}\right)$ as

$$
v_{L-1}(w)=\max \left\{w-\frac{\eta-\beta \bar{w}}{1-\beta}+V_{L}(\eta), \bar{e}+V_{L}((1-\beta)(w-\bar{e})+\beta \bar{w})\right\} .
$$

\footnotetext{
${ }^{33}$ Such $w$ 's might not exist for some combination of the parameters. However, in this Proposition we assume that they do $\left(\tau<w^{*}<\bar{w}\right)$. This is in order to consider the most general case.

${ }^{34}$ Again, by the assumption made in this Proposition such $w^{\prime} s$ exist.

${ }^{35} \mathrm{By}$ the assumption of this Proposition $\tau<w_{*}=w^{*}-\bar{e}$. Thus, at $w=w^{*}$ it is possible to exert full effort $\bar{e}$ resulting in $w^{\prime}=\eta$ for $e=\bar{e}$.

${ }^{36}$ The last claim is due to the fact that $V_{L}(w)$ is weakly increasing and continuous with slopes no more than 1 for $w<\eta$.
} 
It is easy to see that $v_{L-1}(w)$ is the maximum of two continuous functions in $w$ where the left function has slope 1 and the right function has slope no more than $1-\beta$. Moreover,

$$
\begin{aligned}
\lim _{w \uparrow w^{*}} w-\frac{\eta-\beta \bar{w}}{1-\beta}+V_{L}(\eta) & =\bar{e}+v_{L}(\eta)+u> \\
>\lim _{w \uparrow w^{*}} \bar{e}+V_{L}((1-\beta)(w-\bar{e})+\beta \bar{w}) & =\bar{e}+v_{L}(\eta)
\end{aligned}
$$

Thus, as $w$ goes to $w_{*}$ the function $w-\frac{\eta-\beta \bar{w}}{1-\beta}+V_{L}(\eta)$ may intersect the function $\bar{e}+V_{L}((1-$ $\beta)(w-\bar{e})+\beta \bar{w}) .{ }^{37}$ If the functions intersect, then we define $a_{L-1}$ as the resource level at the intersection. Below $a_{L-1}$ the optimal choice is $\bar{e}$. If the functions do not intersect then we define $a_{L-1}=w_{*}$ since we know from the above that below $w_{*}$ the optimal choices are like in Proposition 3. The alternative representation, (15) also makes it easy to see that $v_{L-1}$ is continuous at $a_{L-1}$ whichever way it is defined.

Combining the above observations, we can conclude that $v_{L-1}(w)$ is a weakly increasing, piecewise linear continuous function. To complete the argument, we must show that the slopes of $v_{L-1}$ do not exceed 1. Indeed, we showed that this is the case for $w \geq w^{*}$ and $w \in\left[\tau, a_{L-1}\right)$. On the interval $\left[a_{L-1}, w^{*}\right]$ we have $v_{L-1}(w)=w-\frac{\eta-\beta \bar{w}}{1-\beta}+V_{L}(\eta)$ which has slope 1 .

Since $v_{L-1}(w)$ is a weakly increasing and continuous with slopes no more than 1 , we can conclude that $V_{L-1}(w)$ takes the same form, apart from the discontinuity at $w=\eta$. Thus, $V_{L-1}(w)$, as well as $V_{L}(w)$, is weakly increasing and continuous with slopes no more than 1 with discontinuity at $w=\eta$. In the proof above only these properties of $V_{L}(w)$ were used. Therefore, by induction, all the functions $V_{1}(w), V_{2}(w), \ldots V_{L-2}(w)$ have similar characteristics and optimal choices.

${ }^{37}$ Whether this intersection occurs depends on the parameters. 


\section{Variables Used in the Regressions}

\begin{tabular}{|c|c|c|}
\hline Variable & Range & Definition \\
\hline exhaustion & {$[1,10]$} & $\begin{array}{l}\text { The number of period in which a group exhausted } \\
\text { the resource }\end{array}$ \\
\hline breaker & $1 / 0$ & $\begin{array}{l}\text { Is } 1 \text { if subject spent less than } 25 \text { seconds waiting at } \\
\text { the traffic lights in the RF task }\end{array}$ \\
\hline $\mathrm{CPRH}$ & $1 / 0$ & Is 1 if CPRH treatment \\
\hline restraint $_{i, t}$ & $1 / 0$ & $\begin{array}{l}\text { For subject } i \text { in period } t \text {. For } w \geq 270 \text { is } 1 \text { if the } \\
\text { effort is less than or equal to } 60 \text {; for } w \in(30,270) \text { is } \\
1 \text { if the choice is less than or equal to }(w-\tau) / n= \\
(w-30) / 4\end{array}$ \\
\hline others $_{\text {restraint }}, t-1$ & $1 / 0$ & $\begin{array}{l}\text { Is } 1 \text { if others showed restraint in the group of } \\
\text { subject } i \text { in period } t-1\end{array}$ \\
\hline Reverse & $1 / 0$ & Is 1 if Reverse treatment \\
\hline
\end{tabular}

Table 3: Variables used in the regressions. 


\section{E Additional Experimental Findings}

\section{E.1 Histograms of Waiting Times}

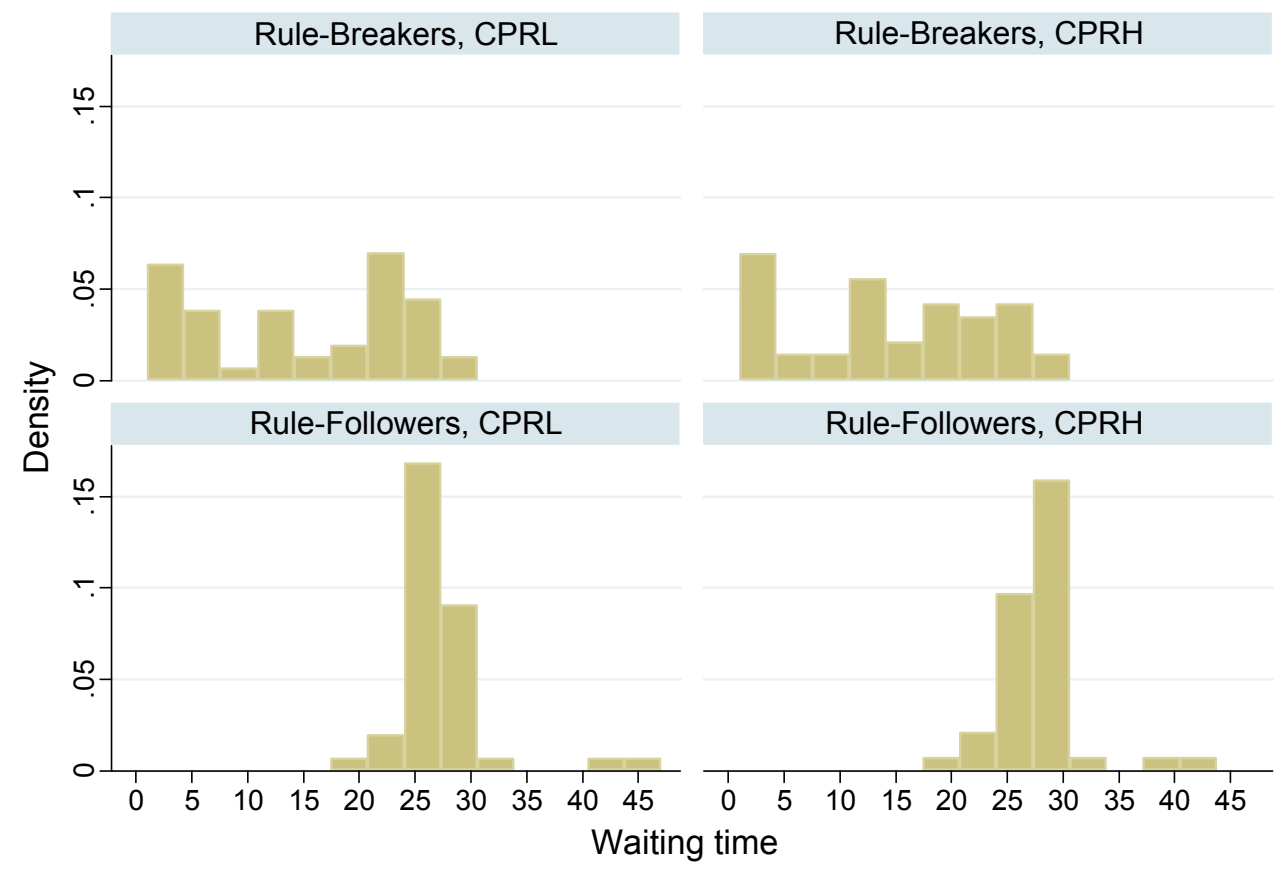

Figure E1: Histograms of total waiting time in RF task by group type in CPRL and CPRH treatments.

\section{E.2 Exponential Growth Treatment}

As an additional control, we explore how resource dynamics depend on the particular choice of resource regeneration function. In the 4 treatments discussed in this article, the resource regeneration function was proportional to the deviation from resource capacity so that the resource grew from level $w$, after harvesting choices were made in period $t$, to $w+\beta(\bar{w}-w)$ at the beginning of period $t+1$. Thus, growth is high for low stocks of the resource and is vanishing as the resource converges to $\bar{w} .{ }^{38}$ We wanted to see how group dynamics would change when we instead introduced an exponential growth function, where total capacity is unbounded. We substituted the "concave" growth above by "convex" one: $w \rightarrow w+\frac{w}{2}$. This growth function has the opposite properties: growth is low for low levels of the resource and high for high levels, and we refer to this as the CPREXP treatment.

Similar to our observations in the CPRH and CPRL treatments, rule-followers wait an average of 26.4 seconds during the RF task, while rule-breakers wait 13.6 seconds on average. Figure E2 displays time series of resource stocks for both rule-followers and rule-breakers

\footnotetext{
${ }^{38}$ This type of growth is inherent to many natural systems, for example, populations of fish. When the fish population is small, there is plenty of food available and population grows very fast. As the population gets very large the growth stops, since there are natural limits on the amount of food available.
} 


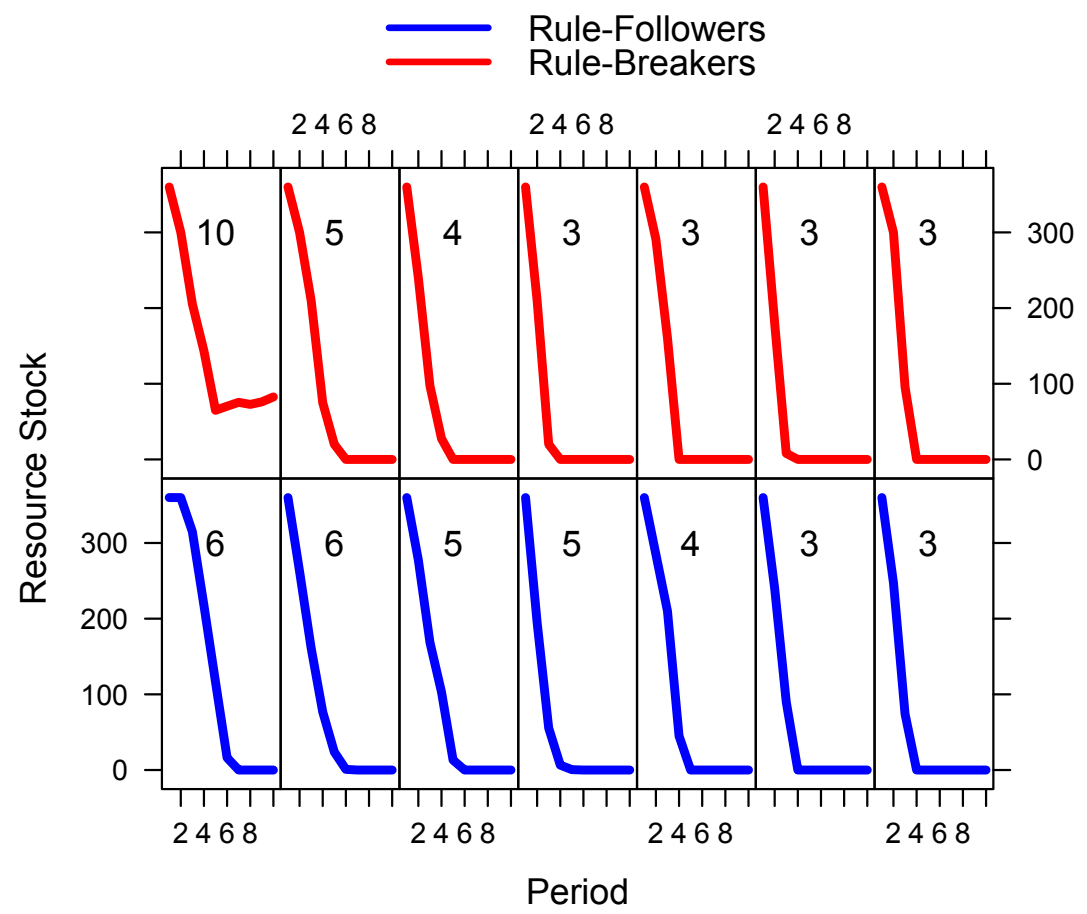

Figure E2: Time series of resource stocks in the CPREXP treatment, for Rule-Followers and Rule-Breakers.

in the CPREXP treatment. With the exception of one group, both rule-following and rulebreaking groups in CPREXP treatment exhaust the resource in about the same number of periods as groups in CPRL treatment (means are 4.57 and 4.42 for followers and breakers, respectively), and permutation tests cannot reject the null hypothesis of equal mean period of exhaustion when comparing either rule-followers or rule-breakers across the treatments ( $p$-values $=1$ and 0.766, two-sided tests). As was discussed above, CPRL groups exhaust the resource relatively quickly because of the lower growth rate as compared to the CPRH treatment. This problem is even more pronounced in the CPREXP treatment since the growth rate declines as the resource stock declines. Thus, we find that rule-followers in the CPREXP treatment exhaust the resource more quickly than their counterparts in the CPRH treatment (two-sided permutation test, $p$-value $=0.019)$. Rule-breakers on the other hand show no significant differences ( $p$-value $=0.392$, two-sided test).

Finding 6. Exponential growth does not encourage groups to sustain the resource because growth is slow when the stock of the resource is small. CPREXP groups are statistically indistinguishable from CPRL groups and rule-breaking CPRH groups, but exhaust the resource significantly faster than rule-following CPRH groups. 


\section{F Instructions for the Rule Following Stage}

\section{General information}

You are now participating in a decision making experiment. If you follow the instructions carefully, you can earn a considerable amount of money depending on your decisions and the decisions of the other participants. Your earnings will be paid to you in CASH at the end of the experiment

This set of instructions is for your private use only. During the experiment you are not allowed to communicate with anybody. In case of questions, please raise your hand. Then we will come to your seat and answer your questions. Any violation of this rule excludes you immediately from the experiment and all payments. The research organization METEOR has provided funds for conducting this experiment.

\section{Part I}

In Part I of this experiment, you control a stick figure that will walk across the screen.

Once the experiment begins, you can start walking by clicking the "Start" button on the left of the screen. Your stick figure will approach a series of stop lights and will stop to wait at each light. To make your stick figure walk again, click the "Walk" button in the middle of the screen.

The rule is to wait at each stop light until it turns green.

Your earnings in Part I are determined by the amount of time it takes your stick figure to walk across the screen. Specifically, you begin with an initial endowment of 8 Euro. Each second, this endowment will decrease by 0.08 Euro.

This is the end of the instructions for Part I. If you have any questions, please raise your hand and an experimenter will answer them privately. Otherwise, please wait quietly for the experiment to begin.

\section{G Instructions for the CPR Game}

\section{Part II}

This part of the experiment will consist of several decision making periods. In each period, you will collect 60 tokens. Your task is to decide whether to take these tokens from either or both of two accounts: a private account and a group account.

Each period you receive the sum of your earnings from your private account plus your earnings from the group account.

There are 4 people, including yourself, participating in your group. You will be matched with the same people for all of Part II. Other people in your group will make the same decisions as you. You share the group account with other members of your group (and only with them and no one else).

Each token you take from the private account generates a cash return to you (and to you alone) of one cent (0.01 Euro).

Tokens taken from the group account yield a different return. For each token you take from 
the group account, you will receive a cash return of two cents (0.02 Euro).

The private account has an unlimited number of tokens that you can take, so it will never run out of tokens. However, the group account initially contains a total of $\mathbf{3 6 0}$ tokens. This is the capacity of the group account.

Whenever any person in your group takes tokens from the group account, the number of tokens is reduced. However, each period, some of the tokens taken from the group account will replenish. Specifically, they will replenish according to the following rule:

If there are $\mathbf{X}$ tokens remaining in the group account at the end of a period, the group account will replenish $(360-X) / 4$ tokens. Thus, at the beginning of the next period, there will be $\mathbf{X}+$ $(360$ - X)/4 tokens in the group account.

BUT if the total number of tokens in the group account ever falls to fewer than $\mathbf{3 0}$ tokens, the group account will not replenish.

Finally, if at any point, the group attempts to take more tokens from the group account than actually remain in the account, the remaining tokens will be divided across the people who chose to take from the group account, and the group account will not replenish.

Here are three examples to make this clear:

(1) Suppose that at the beginning of the period, there are $\mathbf{3 6 0}$ tokens in the group account. Then, suppose the people in your group, including yourself, take a total of $\mathbf{2 0 0}$ tokens from

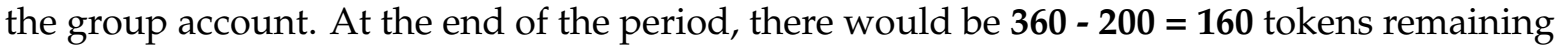
in the group account.

The group account would then replenish before the next period. Specifically, $(360-160) / 4=$ 50 tokens would be added back to the group account.

So, at the beginning of the next period, there would be $\mathbf{1 6 0}+\mathbf{5 0}=\mathbf{2 1 0}$ tokens in the group account.

(2) Now, suppose the next period begins with 210 tokens in the group account, and suppose that the people in your group take $\mathbf{2 0 0}$ tokens from the group account. At the end of the period, there would be $(\mathbf{2 1 0}-\mathbf{2 0 0})=\mathbf{1 0}$ tokens in the group account.

However, since $\mathbf{1 0}<\mathbf{3 0}$ the group account would not replenish, and there would only be 10 tokens in the group account at the beginning of the next period.

(3) Now, suppose the next period begins with $\mathbf{1 0}$ tokens in the group account. Then suppose that one person in the group attempts to collect 10 tokens from the group account, one person attempts to collect 2 tokens, and the other two people only collect from the private account.

Since there are only 10 tokens to collect, the two people who attempted to take from the group account would split the tokens according to the following rule. The first person would collect 1 token, and the second person would collect 1 token. Then the first person would collect another token, and so would the second person. Now the second person has collected all the tokens he/she chose to collect, so the first person would collect the remaining 6 tokens. In this case, the first person would get a total of $\mathbf{8}$ tokens from the group account, and the second person would get 2 tokens. They would then collect the rest of their tokens from the private account.

Each period proceeds as follows:

First, decide on the number of tokens to take from the private and the group accounts by 
entering numbers into the boxes labeled private and group. Your entries must sum to 60.

While you make your decision, the 3 other members in your group will also decide on how many tokens to take from the private and group accounts.

Second, after everyone has made a decision, your earnings for that decision period are the sum of your earnings from the private and group accounts.

As an example, suppose that you take a total of $\mathbf{3 0}$ tokens from the private account and $\mathbf{3 0}$ tokens from the group account. Your total earnings from that period would be $30+30^{*} \mathbf{2}=$ 90 cents. Remember, the return from the group account is $\mathbf{0 . 0 2}$ Euro per token and the return from the private account is $\mathbf{0 . 0 1}$ Euro per token.

While you are deciding how to allocate your tokens, everyone else in your group will be doing so as well. When the period is over the computer will display your earnings for that period and your total earnings up to and including that period.

This is the end of the instructions. If you have any questions please raise your hand and an experimenter will come by to answer them. 